\title{
Analyzing Tropical Cyclone Structures during Secondary Eyewall Formation Using Aircraft In Situ Observations
}

\author{
Katharine E. D. Wunsch and Anthony C. Didlake JR. \\ Department of Meteorology and Atmospheric Science, The Pennsylvania State University, University Park, Pennsylvania
}

(Manuscript received 1 June 2018, in final form 10 September 2018)

\begin{abstract}
The dynamical mechanisms for secondary eyewall formation (SEF) in tropical cyclones (TCs) are not yet fully understood. Most hypotheses for SEF rely on the early presence of persistent and widespread rainband convection outside of the primary eyewall. This convection eventually coalesces into a secondary eyewall through both axisymmetric and asymmetric processes, but the extent and importance of these dynamical processes and their associated convective structures remain unclear. This study examines the evolution of axisymmetric and asymmetric structures in a composite analysis of Atlantic TCs from 1999 to 2015 using aircraft reconnaissance observations from the Extended Flight-Level Dataset for Tropical Cyclones (FLIGHT+). Compared to intensifying TCs that did not experience SEF, TCs undergoing SEF showed axisymmetric broadening of the outer wind field in the tangential wind and angular momentum profiles before SEF. Thermodynamic observations indicated features consistent with strengthening eyewall convection. We also analyzed TCs in shear-relative quadrants to examine the evolution of asymmetric kinematic and thermodynamic structures during SEF. Utilizing a new normalization technique based on the radii of both eyewalls, we isolated the structures surrounding the secondary eyewall before and during SEF. Using this technique, we found that kinematic structures of the developing secondary eyewall were most prominent in the left-of-shear half, and the thermodynamic structures of the secondary eyewall became more axisymmetric during SEF. Asymmetries developed in the primary eyewall thermodynamics as it decayed. Understanding the evolution of these observed structures characteristic to SEF will improve our ability to predict SEF and the resulting changes in TC intensity and structure.
\end{abstract}

\section{Introduction}

The intensity and structure of tropical cyclones (TCs) are influenced by both large-scale environmental factors and the evolution of inner-core features (e.g., Gray 1968; Houze 2010). In some intense TCs, the rainbands in the inner core can coalesce and form into a secondary eyewall with an associated tangential wind maximum outside of the preexisting eyewall (Willoughby et al. 1982; Black and Willoughby 1992; Dodge et al. 1999; Didlake and Houze 2011; Bell et al. 2012). The formation of a secondary eyewall often triggers an eyewall replacement cycle (ERC), in which characteristic changes in intensity and structure occur (Sitkowski et al. 2011, 2012).

The exact processes that cause secondary eyewall formation (SEF) are not yet clear. Several theories describe potential formation mechanisms, which involve critical TC processes such as vortex Rossby wave propagation and stagnation (Montgomery and Kallenbach 1997),

Corresponding author: Anthony C. Didlake Jr., didlake@psu.edu upscale cascade of vorticity anomalies along a sufficient radial vorticity gradient (Terwey and Montgomery 2008), or axisymmetric boundary layer dynamics that drive a positive feedback between convection and the growing secondary wind maximum (Kepert 2013; Huang et al. 2012; Abarca and Montgomery 2013; Zhang et al. 2017). Observations and modeling studies regularly find that prior to SEF, the outer wind field of the TC expands (e.g., Sitkowski et al. 2011, 2012; Didlake and Houze 2013a,b; Qiu and Tan 2013; Sun et al. 2013; Wang et al. 2016; Tang et al. 2017; Zhang et al. 2017). Some studies identify this broadened wind field as a critical component for SEF (Rozoff et al. 2006, 2012; Fang and Zhang 2012). However, it is not clear if this wind field expansion is a catalyst for SEF, or if it is a result of SEF processes that have already begun.

Despite the varying SEF hypotheses, most theories indicate that the inner-core rainbands are crucial for SEF, where rainband convection and the associated vorticity structures must sufficiently project onto the azimuthal mean. Just prior, rainband convection consists of 
asymmetric structures that may have an earlier impact on eventual SEF. Under environmental wind shear, the rainband complex tends to be oriented such that active convective cells inhabit the TC half that lies to the right of the deep-layer environmental wind shear vector. Meanwhile, slowly falling ice crystals are advected downwind and fall out as stratiform precipitation in the left-of-shear half of the storm (Black et al. 2002; Hence and Houze 2012b; Didlake and Houze 2013a,b). Several studies consider the downwind stratiform portions of the rainband complex to be instrumental in the formation of the secondary eyewall (Qiu and Tan 2013; Fang and Zhang 2012; Didlake and Houze 2013b; Dai et al. 2017; Zhang et al. 2017; Didlake et al. 2018). These rainband features project more strongly onto the axisymmetric structure than do the convective rainbands upwind, given both the mesoscale, homogenous nature of stratiform precipitation and the fact that these features occur at a smaller radius on the downwind end of an inward spiraling complex.

Didlake and Houze (2013b) found that mesoscale descending inflow occurred in the stratiform region of a rainband complex, and they postulated that this flow pattern may have an effect on SEF. In their findings, latent heating and cooling patterns within the stratiform rainband created horizontal buoyancy gradients that induced a descending inflow pattern similar to that of the trailing stratiform region of a mesoscale convective system (Parker and Johnson 2000; Houze 2004). The negatively buoyant descending air then extended into the boundary layer, similar to that simulated by Qiu and Tan (2013). Didlake et al. (2018) argued that this negatively buoyant downdraft induces upward acceleration and convergence within the boundary layer, creating persistent deep convection that ultimately leads to SEF. Since stratiform rainband precipitation is preferred in the left-of-shear half of the storm, the mesoscale descending inflow and nascent secondary eyewall convection are also expected in this storm region (Didlake et al. 2017).

These SEF theories all describe an evolution of characteristics that are distinguishable traits of storms developing a secondary eyewall. However, the initial stages of these theories involve characteristics that can exist in storms that do not undergo SEF (e.g., vortex Rossby wave propagation or ample rainband activity). The extent to which these evolving features become unambiguous indicators of SEF is not apparent in the current literature. The current study explores this problem by using storm composites to seek structural characteristics, both axisymmetric and asymmetric, that typify and help explain SEF processes. Building upon results from Sitkowski et al. (2011, 2012), we composite aircraft reconnaissance 700-hPa-level observations from
ERC events prior to and during the formation of the secondary eyewall. We first compare axisymmetric structures of ERC storms to those of strong and intensifying TCs that did not undergo ERCs. We focus on strong and intensifying TCs since SEF tends to occur more frequently in stronger TCs, often following a period of intensification (Hawkins and Helveston 2008; Zhang et al. 2017). This portion of our methodology is based on that of Martinez et al. (2017), who compared intensifying, steady-state, and weakening TCs using a composite analysis of flight-level data from reconnaissance aircraft. We then analyze the evolution of asymmetric structures in ERC storms to identify additional distinguishing features for SEF. The asymmetric analysis is defined by the deep-layer environmental wind shear. The kinematic and thermodynamic characteristics identified in this two-part study will be examined in the context of hypothesized SEF mechanisms. Section 2 outlines the data sources and methods by which data were processed and analyzed. Section 3 analyzes the axisymmetric structural differences between TCs undergoing SEF and non-SEF TCs. Section 4 investigates the TC asymmetric structural evolution during SEF, and section 5 presents the conclusions from the current study.

\section{Data and methodology}

\section{a. Flight-level data and processing techniques}

The primary data source for this study is the Extended Flight-Level Dataset for Tropical Cyclones (FLIGHT+; Vigh et al. 2016). This dataset contains flight-level in situ observations of TCs from all National Oceanic and Atmospheric Administration WP-3D and U.S. Air Force C-130 reconnaissance missions during 1999-2015. These aircraft typically fly in figure- 4 or butterfly patterns to gather multiple transects across the storm. FLIGHT+ organizes these observations into radial legs using the storm centers determined by the Willoughby and Chelmow (1982) method. For this analysis, we included radial legs where the aircraft flew within $25 \mathrm{~km}$ of the storm center of the TC and had a radial extent of at least $45 \mathrm{~km}$ (these are the "good radial legs" in FLIGHT + ). Only flight legs that occurred at the 700-hPa level were considered in this study. Data from the radial legs were first interpolated to a radial grid with a resolution of $0.5 \mathrm{~km}$ and extending out to $350 \mathrm{~km}$. To reduce noise in the observations, a 10-km Lanczos filter was applied (Duchon 1979), following the methodology of Martinez et al. (2017). Next, radial legs from each flight were organized into "sets" of 4-7 legs, either inbound or outbound, that were flown consecutively. This means that, for example, a flight with only three radial legs would 
a)

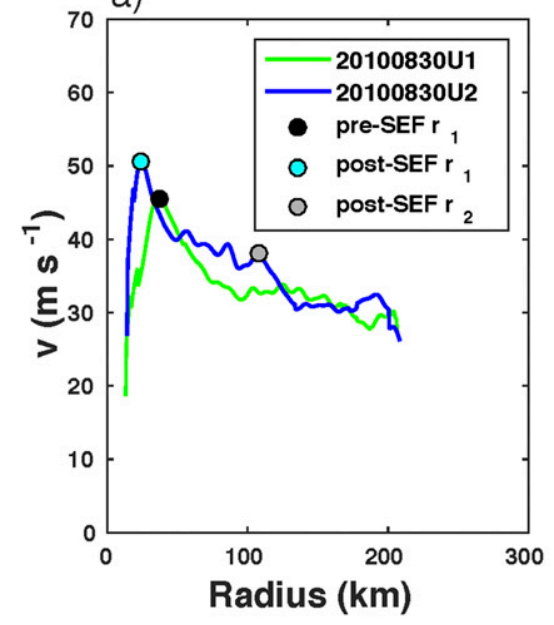

b)

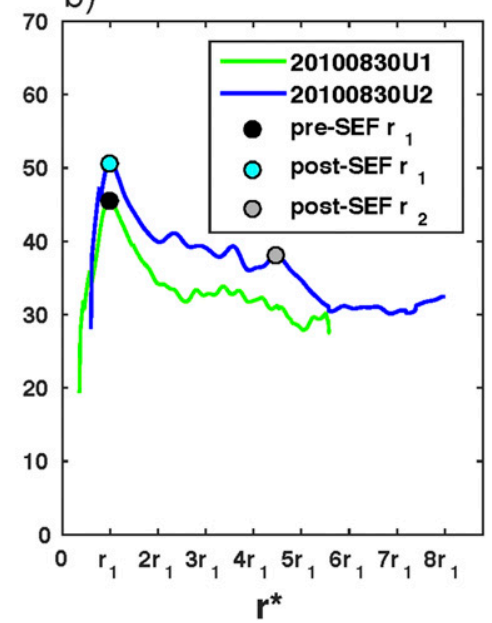

C)

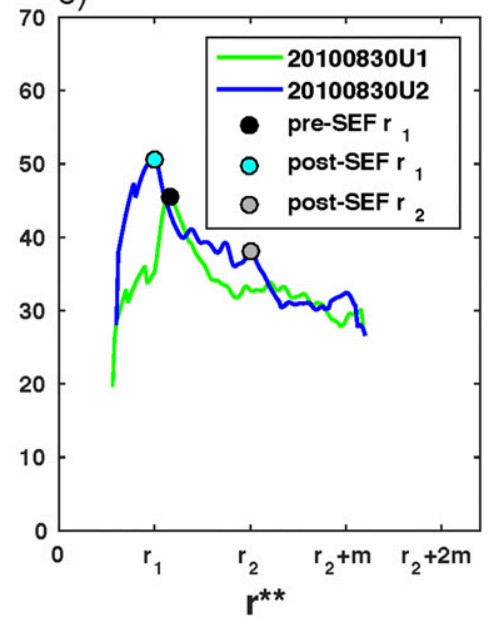

FIG. 1. Set-mean tangential wind profiles from flight missions 20100830U1 and 20100830U2 into Hurricane Earl. (a) Profiles along true radius $(\mathrm{km})$. The radius of maximum winds is denoted by black and cyan dots for 20100830U1 and 20100830U2, respectively. The radius of the secondary wind maximum is noted by a gray dot in 20100830U2. (b) Profiles along radius normalized by the radius of maximum wind $\left(r^{*}\right)$ in each set. (c) Profiles along radius normalized by the radii of both the primary wind maximum and secondary wind maximum $\left(r^{* *}\right)$ at the time when the secondary eyewall is first observed.

not have been included; a flight with five radial legs constituted one set; and a flight with eight radial legs was divided into two sets, where each set included four consecutive legs. Most of the sets contained legs evenly spaced in azimuth around the storm, providing an azimuthal mean representative of the axisymmetric storm structure. Some sets had large azimuthal gaps between legs due to an irregular flight pattern. Removal of these sets from the analysis did not affect the overall conclusions of the study. To correct for instrument wetting, the method described by Zipser et al. (1981) was applied to reduce errors in temperature and dewpoint observations; this method is consistent with previous studies using aircraft observations of TCs.

\section{b. Group classification}

ERC "events" in the North Atlantic were identified by first examining the azimuthal-mean tangential velocity of each set. We applied an algorithm that located local maxima in the set-mean wind field. This MATLAB algorithm (findpeaks) took into account the width and relative magnitude of each peak to capture the primary eyewall peak and meaningful signals of potential secondary eyewall formation. The value of each parameter was adjusted subjectively to ensure that the maxima being captured were associated with the eyewalls; moderate changes in these parameters did not yield changes in the overall conclusions of this study. Next, we used a combination of datasets to confirm that each set of flight legs with an identified secondary wind maximum was actually part of an ERC event observed over time. These datasets included microwave satellite imagery, airborne radar imagery, National Hurricane Center (NHC) storm reports, and the current FLIGHT+ tangential wind mean profiles. We examined the potential cases for specific indicators of an ERC: a contracting axisymmetric secondary ring of convection, a contracting axisymmetric secondary wind maximum, or the decay of inner eyewall convection. If any of these features were identified, then these cases were confirmed to be an ERC event.

Figure 1a shows an example of two consecutive sets from a confirmed ERC in Hurricane Earl (2010). In the first set, only the primary wind maximum was identified and is labeled as $r_{1}$, while the next set had the first appearance of a secondary wind maximum, which is labeled as $r_{2}$. The set with the first appearance of the secondary wind maximum is called the "anchor" set of the event. The next step was to define the time span of each ERC event. Particularly, we identified the beginning time of each event to determine those sets that preceded the anchor set and captured the secondary eyewall formation process. To do this, we examined the evolution of the storm's central pressure during each event using the NHC best track dataset. An ERC is typically accompanied by a fluctuation in the central pressure. For each event, we identified the local minimum pressure that occurred near the time of the anchor set, and this time of the minimum pressure was noted. The beginning time of each event was defined as $36 \mathrm{~h}$ prior to the time of the minimum pressure. Flight missions (and their associated sets) that took place 
TABLE 1. Selected eyewall replacement cycle events in Atlantic TCs from 1999 to 2015 that coincide with aircraft flight missions. Flight IDs are in a format that includes the date (yyyymmdd) and identification code for the specific mission tasked by each agency.

\begin{tabular}{|c|c|c|c|}
\hline $\begin{array}{l}\text { Storm name-ERC } \\
\text { event number }\end{array}$ & Year & Flight ID (total number of radial legs) & $\begin{array}{l}\text { Pre-SEF group set counts, } \\
\text { post-SEF group counts }\end{array}$ \\
\hline Floyd-1 & 1999 & $\begin{array}{l}\text { 19990910U2 (7), 19990911U1 (8), } 19990911 \mathrm{U} 2 \text { (4), 19990912U1 (8), } \\
\text { 19990912U2 (10) }\end{array}$ & 2,1 \\
\hline Floyd-2 & 1999 & $\begin{array}{l}\text { 19990912U1 (8), 19990912U2 (10), 19990913U1 (8), 19990913I1 (8), } \\
\text { 19990914U1 (6), 19990914U2 (9), 19990914U3 (7) }\end{array}$ & 6,2 \\
\hline Gert-1 & 1999 & $19990916 \mathrm{U} 1(4), 19990916 \mathrm{U} 2(4)$ & 1,1 \\
\hline Erin-1 & 2001 & 20010909_AF980_0706A (8), 20010909_AF985_0806A (7) & 1,1 \\
\hline Michelle-1 & 2001 & $\begin{array}{l}\text { 20011103_AF966_1015A (7), 20011103_AF861_1115A (5), 20011103H1 } \\
(10), 20011104 \mathrm{H} 1 \text { (10), 20011104H2 (6) }\end{array}$ & 0,1 \\
\hline Isidore-1 & 2002 & 20020920U1 (7), 20020920U2 (10), $20020921 \mathrm{U} 1$ (8), 20020922U1 (7) & 0,1 \\
\hline Fabian-1 & 2003 & $\begin{array}{l}20030902 \mathrm{I} 1 \text { (5), 20030902H1 (5), } 20030903 \mathrm{U} 1 \text { (4), } 20030903 \mathrm{U} 2 \text { (4), } \\
\text { 20030904U3 (4), 20030905U1 (8) }\end{array}$ & 2,2 \\
\hline Isabel-1 & 2003 & $\begin{array}{l}\text { 20030916U2 (8), 20030917U1 (7), } 20030917 \mathrm{U} 2 \text { (6), 20030917U3 (9), } \\
\text { 20030918U1 (7) }\end{array}$ & 0,0 \\
\hline Frances-1 & 2004 & $\begin{array}{l}\text { 20040830u1 (4), 20040830I1 (6), 20040831u1 (8), 20040831I1 (4), } \\
\text { 20040831u2 (7), 20040901u1 (7) }\end{array}$ & 0,1 \\
\hline Frances-2 & 2004 & $\begin{array}{l}20040830 \mathrm{I} 1(6), 20040831 \mathrm{u} 1(8), 20040831 \mathrm{I} 1(4), 20040831 \mathrm{u} 2(7) \\
20040901 \mathrm{u} 1(7), 20040901 \mathrm{I} 1(6), 20040901 \mathrm{u} 2(10), 20040902 \mathrm{u} 1(10) \\
20040902 \mathrm{u} 2(6)\end{array}$ & 5,1 \\
\hline Frances-3 & 2004 & $\begin{array}{l}20040831 \mathrm{I} 1(4), 20040831 \mathrm{u} 2(7), 20040901 \mathrm{u} 1(7), 20040901 \mathrm{I} 1 \text { (6), } \\
20040901 \mathrm{u} 2(10), 20040902 \mathrm{u} 1(10), 20040902 \mathrm{u} 2(6), 20040902 \mathrm{u} 3(9) \\
20040903 \mathrm{u} 1(4), 20040903 \mathrm{I} 1(5), 20040903 \mathrm{u} 2(4)\end{array}$ & 2,1 \\
\hline Frances-4 & 2004 & $\begin{array}{l}20040902 \mathrm{u} 2(6), 20040902 \mathrm{u} 3(9), 20040903 u 1(4), 20040903 \mathrm{I} 1 \text { (5), } \\
20040903 \mathrm{u} 2(8), 20040903 \mathrm{u} 3(10), 20040904 \mathrm{u} 1(9), 20040904 \mathrm{u} 2(8)\end{array}$ & 1,1 \\
\hline Ivan-1 & 2004 & $\begin{array}{l}20040908 \mathrm{u} 1(7), 20040908 \mathrm{u} 2(8), 20040909 \mathrm{u} 1 \text { (9), } 20040909 \mathrm{I} 1 \text { (6), } \\
20040909 \mathrm{u} 2(8), 20040910 \mathrm{u} 1(10), 20040910 \mathrm{u} 2(9)\end{array}$ & 3,1 \\
\hline Ivan-2 & 2004 & $\begin{array}{l}20040909 \mathrm{I} 1 \text { (6), 20040909u2 (8), 20040910u1 (10), 20040910u2 (9), } \\
20040911 \mathrm{u} 1(6), 20040911 \mathrm{u} 2(6), 20040912 \mathrm{u} 1(8), 20040912 \mathrm{I} 1 \text { (6), } \\
20040912 \mathrm{u} 2(6), 20040912 \mathrm{H} 1(4)\end{array}$ & 1,1 \\
\hline Ivan-3 & 2004 & $\begin{array}{l}20040910 u 2(9), 20040911 u 1(6), 20040911 u 2(10), 20040912 u 1(8), \\
20040912 \mathrm{I} 1(6), 20040912 \mathrm{u} 2(6), 20040912 \mathrm{H} 1(8), 20040912 \mathrm{u} 2(6)\end{array}$ & 0,1 \\
\hline Ivan-4 & 2004 & $\begin{array}{l}20040912 \mathrm{u} 1(4), 20040912 \mathrm{I} 1(6), 20040912 \mathrm{u} 2(6), 20040912 \mathrm{H} 1(8) \\
20040913 \mathrm{u} 1(7), 20040913 \mathrm{u} 2(5), 20040913 \mathrm{H} 1(7), 20040913 \mathrm{I} 1 \text { (4), } \\
20040913 \mathrm{u} 3(4), 20040914 \mathrm{u} 1(10), 20040914 \mathrm{H} 1(13), 20040914 \mathrm{u} 3(5) \\
20040914 \mathrm{I} 1(9), 20040915 \mathrm{u} 2(4)\end{array}$ & 1,1 \\
\hline Katrina-1 & 2005 & $\begin{array}{l}\text { 20050826U1 (10), 20050827U1 (10), 20050827I1 (5), 20050827U2 (6), } \\
\text { 20050827U3 (8), 20050828U1 (10), 20050828I1 (10) }\end{array}$ & 3,1 \\
\hline Katrina-2 & 2005 & $\begin{array}{l}\text { 20050827U1 (10), 20050827I1 (5), 20050827U2 (6), 20050827U3 (8), } \\
\text { 20050828U1 (10), 20050828I1 (10), 20050829U2 (11), 20050829U3 (9) }\end{array}$ & 1,3 \\
\hline Rita-1 & 2005 & $\begin{array}{l}20050920 \mathrm{U} 1(8), 20050920 \mathrm{U} 2(10), 20050921 \mathrm{U} 1(8), 20050922 \mathrm{U} 1 \text { (8), } \\
20050922 \mathrm{I} 1 \text { (10), 20050922U2 (7), 20050923U1 (8) }\end{array}$ & 2,1 \\
\hline Wilma-1 & 2005 & 20051019U2 (5), 20051020U1 (7), 20051020H1 (8) & 0,1 \\
\hline Dean-1 & 2007 & $\begin{array}{l}\text { 20070817U1 (9), 20070817U2 (6), } 20070818 \mathrm{U} 1 \text { (10), 20070818U2 (11), } \\
\text { 20070819U2 (7) }\end{array}$ & 3,1 \\
\hline Dean-2 & 2007 & $\begin{array}{l}\text { 20070817U2 (6), 20070818U1 (10), } 20070818 \mathrm{U} 2(11), 20070819 \mathrm{U} 2 \text { (7), } \\
\text { 20070819U3 (8), 20070820U2 (6) }\end{array}$ & 1,1 \\
\hline Felix-1 & 2007 & $\begin{array}{l}\text { 20070902U1 (5), 20070902U2 (4), } 20070903 \mathrm{U} 1 \text { (4), } 20070903 \mathrm{U} 3 \text { (6), } \\
\text { 20070904U1 (6) }\end{array}$ & 3,2 \\
\hline Ike-1 & 2008 & $\begin{array}{l}\text { 20080909U2 (5), 20080910U1 (10), 20080910U2 (7), 20080911U1 (8), } \\
\text { 20080911U2 (8), 20080912U1 (10), 20080912U2 (4) }\end{array}$ & 1,2 \\
\hline Bill-1 & 2009 & $\begin{array}{l}\text { 20090820U1 (4), 20090820U2 (4), } 20090821 \mathrm{U} 1 \text { (4), 20090821U2 (4), } \\
\text { 20090822U1 (4) }\end{array}$ & 2,1 \\
\hline Danielle-1 & 2010 & 20100827U1 (4), 20100828U1 (4) & 0,1 \\
\hline Earl-1 & 2010 & $\begin{array}{l}\text { 20100829U2 (6), 20100830U1 (7), } 20100830 \mathrm{U} 2 \text { (9), 20100831U1 (5), } \\
\text { 20100901U1 (4) }\end{array}$ & 2,1 \\
\hline Earl-2 & 2010 & $\begin{array}{l}\text { 20100901U1 (4), 20100902U1 (7), } 20100902 \mathrm{U} 2 \text { (8), 20100902U3 (8), } \\
\text { 20100903U2 (7) }\end{array}$ & 1,1 \\
\hline Igor-1 & 2010 & 20100916U2 (4), 20100917U2 (4) & 1,0 \\
\hline
\end{tabular}


TABLE 1. (Continued)

\begin{tabular}{cccc}
\hline \hline $\begin{array}{c}\text { Storm name-ERC } \\
\text { event number }\end{array}$ & Year & Flight ID (total number of radial legs) & $\begin{array}{c}\text { Pre-SEF group set counts, } \\
\text { post-SEF group counts }\end{array}$ \\
\hline Irene-1 & 2011 & 20110823U2 (4), 20110823U3 (6), 20110824U1 (8), 20110824U2 (8), & 3,1 \\
& & 20110825U1 (8), 20110825U2 (7), 20110826U1 (8), 20110826U2 (8) & 3,1 \\
Irene-2 & 2011 & $\begin{array}{r}\text { 20110824U2 (8), 20110825U1 (8), 20110825U2 (7), 20110826U1 (4), } \\
\text { 20110826U2 (8), 20110826U3 (8), 20110827U1 (8), 20110827U2 (4) }\end{array}$ & 3,1 \\
Gonzalo-1 & 2014 & 20141014U2 (8), 20141014U3 (6), 20141015U1 (6), 20141015U3 (6) & 3 \\
\hline
\end{tabular}

within $36 \mathrm{~h}$ of the minimum pressure time initially defined an ERC event. Those events were then refined based on the other datasets (i.e., satellite and radar imagery), and any overlap between successive ERC events was removed (discussed further later in this section).

Atlantic basin storms that undergo an ERC tend to be major (category 3 or higher) hurricanes (Hawkins et al. 2006; Kossin and Sitkowski 2009). In this study, fewer than $10 \%$ of identified ERC events occurred in storms below major hurricane status. We, therefore, only focus on sets when the maximum wind speed of the storm reached a minimum of $96 \mathrm{kt}\left(49.4 \mathrm{~m} \mathrm{~s}^{-1}\right)$ in the NHC best track dataset at some point during the set time span. Weaker intensities may have an unknown impact on the structural evolution of the storm during ERCs; effects due to this possibility are now avoided. This methodology resulted in $32 \mathrm{ERC}$ events in 21 North Atlantic TCs listed in Table 1. The identified ERC events included all of the events analyzed by Sitkowski et al. $(2011,2012)$ that are in the FLIGHT+ database. Table 1 also shows the minimum pressure time, the flight IDs for each mission, and the number of radial legs in each mission.

The sets of flight legs in each ERC event were categorized into two "groups" that best capture the evolution of structures leading up to secondary eyewall formation. The post-SEF group consisted of all anchor sets and any set that occurred during the same flight as an anchor set and had a secondary tangential wind maximum. The pre-SEF group consisted of all other sets that occurred before the anchor set and after the beginning time of the event. The beginning time of the final leg of each set was used for this time comparison. Some TCs had successive ERC events, which affected some sets in the pre-SEF group. For these ERC events, the beginning time of an event often extended into the ending stage of the previous event. Therefore, the end of an event was marked by the decay of an inner eyewall wind maximum. To account for this overlap, we removed sets from the pre-SEF group that had local maxima in the tangential wind profile radially inward of the maximum wind. The final total in each group was 33 pre-SEF sets and 30 post-SEF sets during major hurricane ERC events. Table 1 lists the number of sets from each event that were part of each group.

We also analyzed storms that did not go through ERCs for comparison with the storms that did. The nonERC sets were those from FLIGHT+ that were not included in the ERC events. Using the NHC best track dataset, we identified how the TC intensity changed over a 12-h period that encompassed the time of the set of flight legs. When the intensity increased by at least $10 \mathrm{kt}\left(5.1 \mathrm{~m} \mathrm{~s}^{-1}\right)$, those sets were selected for the intensifying (IN) group. This is the same criterion used by Martinez et al. (2017). As with the two ERC groups, only major hurricanes were considered for the IN group. The resultant storms, flight numbers, and number of sets from the IN group are listed in Table 2.

\section{c. Radius normalization techniques}

With such a wide variety of storm sizes and structures, the storm data must be analyzed in a way that highlights

TABLE 2. Selected intensifying, non-ERC Atlantic TCs from 1999 to 2012 that coincide with aircraft flight missions. Flight ID format is as in Table 1.

\begin{tabular}{lllr}
\hline \hline Storm name & Year & \multicolumn{1}{c}{ Flight ID (total number of radial legs) } & No. of sets \\
\hline Bret & 1999 & 19990821U2 (6), 19990822U2 (10) & 3 \\
Lenny & 1999 & 19991117U1 (6), 19991117U3 (8) & 3 \\
Keith & 2000 & $20001001 \mathrm{U} 1(6)$ & 1 \\
Lili & 2002 & $20021002 \mathrm{U} 1(10)$ & 2 \\
Jeanne & 2004 & $20040925 \mathrm{u} 1(9)$ & 4 \\
Dennis & 2005 & $20050707 \mathrm{U} 2(11), 20050709 \mathrm{U} 3(11)$ & 5 \\
Emily & 2005 & $20050714 \mathrm{U} 2(7), 20050715 \mathrm{U} 1(10), 20050716 \mathrm{U} 1(8)$ & 1 \\
Paloma & 2008 & $20081108 \mathrm{U} 1(6)$ & \\
\hline
\end{tabular}


fundamental structures across all storms. To do this, we normalize the radial dimension of the set profiles using two distinct methods. The first method follows Martinez et al. (2017) and previous studies by identifying the radius of maximum wind $\left(r_{1}\right.$; in the gridded radial legs of $0.5-\mathrm{km}$ resolution) from the azimuthal mean of each set. The radial axis is then incremented by $r_{1} / 50$ for each set and extended out to 8 times $r_{1}$. Figure $1 \mathrm{~b}$ shows our example sets in this normalized radius coordinate that we call the $r^{*}$ coordinate space. The $r^{*}$ coordinate normalization is applied to the preSEF, post-SEF, and IN groups in section 3. Figure 2 displays the distribution of the $r_{1}$ values for these three groups.

In addition to variation with the radius of maximum wind, the radius of the secondary wind maximum can vary widely. Of particular interest is the distance between $r_{1}$ and $r_{2}$, known as the moat region, which increasingly exhibits characteristics of an eye during ERCs (Houze et al. 2007). Figure 2 also shows the distributions of $r_{1}, r_{2}$, and the moat width (distance between $r_{1}$ and $\left.r_{2} ; m\right)$ for the ERC anchor sets. Values of $m$ range from 40 to $120 \mathrm{~km}$, with a median of $92 \mathrm{~km}$. One goal of this study is to examine structures that lead up to SEF. Given the large variance of $m$, the $r^{*}$ coordinate space would not be effective for the pre-SEF and post-SEF groups because a composite could obscure those signatures in individual sets that occur on either side of the secondary wind maximum at $r_{2}$. We, therefore, apply a new normalization technique based on both $r_{1}$ and $r_{2}$, hereafter referred to as $r^{* *}$. In this $r^{* *}$ coordinate space, the normalization points along the radial axis begin with $r_{1}$ and $r_{2}$ from each anchor set. The normalization points continue out from the secondary wind maximum $\left(r_{2}\right)$ at distances equal to $m$ and $2 m$. Thus, we are creating a radial coordinate system that first goes from 0 to $r_{1}$ in increments of $r_{1} / 50$. Continuing outward, the radial coordinate then is uniformly spaced from $r_{1}$ to $r_{2}, r_{2}$ to $r_{2}+m$, and $r_{2}+m$ to $r_{2}+2 m$, with increments of $m / 50$. The same normalized radius coordinate for the anchor set was used for all sets within that event to track the ERC evolution. Figure 1c shows our example of pre- and post-SEF sets normalized into $r^{* *}$ space for two sets during Hurricane Earl. This normalization scheme is utilized for the analysis in sections 4 and 5 .

Also in section 4, we examine each radial leg relative to the large-scale environmental wind shear. The wind shear used in this study is the $850-200-\mathrm{hPa}$ shear from the SHIPS analysis database (DeMaria et al. 2005). These 6-hourly shear vectors are linearly interpolated to the center time of each radial leg. An average azimuth is then calculated for each radial leg and assigned to the shear quadrant [downshear left (DL), downshear right

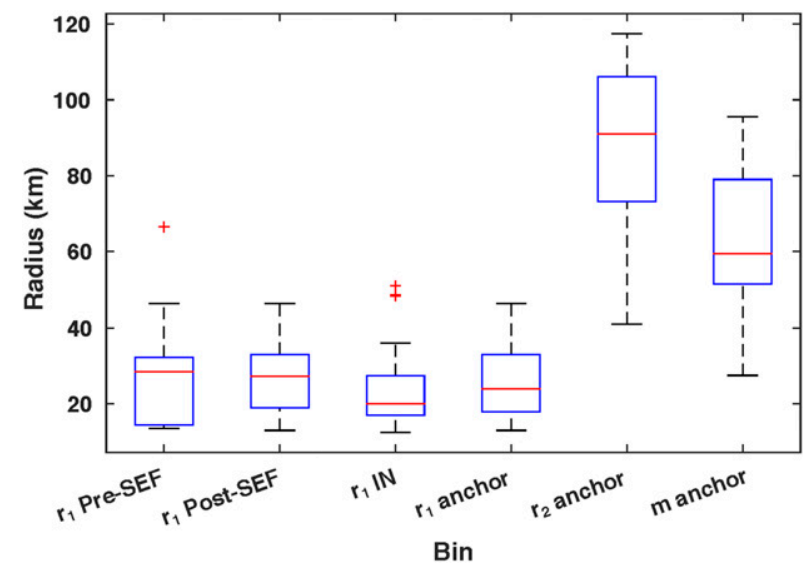

FIG. 2. Box-and-whisker plot showing distributions of the radius normalization parameters. The radius of maximum wind $r_{1}$ is shown for the pre-SEF, post-SEF, and IN groups. The radius of maximum wind, radius of secondary wind maximum $r_{2}$, and moat radial distance $m$ are shown for the anchor sets, which is when the secondary eyewall is first observed in each event.

(DR), upshear left (UL), and upshear right (UR)] based on the shear direction at that time.

Finally, the radial legs may not have data at all radii. To avoid unrepresentative mean profiles, a minimum data count threshold was implemented at each radius. Each group must have data for at least three sets in $r^{*}$ space, and each quadrant must have data for at least 10 legs in $r^{* *}$ space. This threshold cuts off some data in the eye and at outer radii.

\section{Axisymmetric composite structures of ERC and non-ERC TCs}

The first analysis compares the kinematic and thermodynamic structures of storms that underwent an ERC to those that did not. Specifically, we examine the mean of the azimuthal set means from the pre-SEF, post-SEF, and IN storm groups in the $r^{*}$ coordinate framework. By comparing the axisymmetric radial profiles of TCs that went through ERCs to those that did not, we can gain an understanding of critical differences between the two storm types and the resulting implications on differences in storm structure. Given that SEF often occurs during or after the intensification of a TC, we can consider the three groups (pre-SEF, post-SEF, and IN) as consecutive moments along the timeline of an evolving storm. In total, 29 of our 33 pre-SEF sets were intensifying or steady state during the observation time span. Comparing the evolution of these profiles can highlight the earliest differences between ERC and non-ERC storms. At each normalized radius, the distributions representing each group were compared: using a two-tailed WilcoxonMann-Whitney rank-sum test, statistically significant 

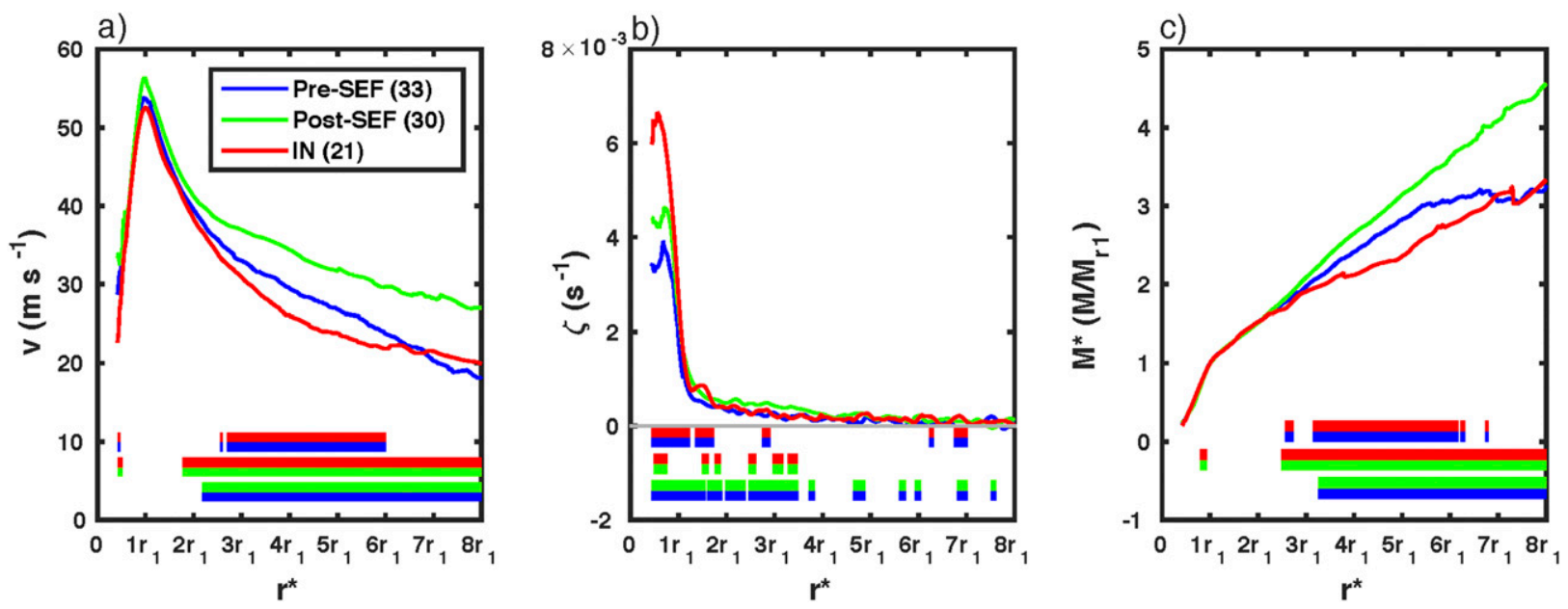

FIG. 3. Composite-mean radial profiles for pre-SEF, post-SEF, and IN groups in $r^{*}$ space (normalized by the radius of maximum wind) for (a) tangential wind, (b) relative vorticity, and (c) nondimensional angular momentum. Statistically significant differences between each group pair are plotted along the bottom of each plot with colors corresponding to each group. Numbers in the legend denote the sample count in each group.

differences at the $95 \%$ confidence interval are calculated for each group pair.

\section{a. Axisymmetric composite-mean kinematic structures}

The most prominent differences in the axisymmetric tangential wind profiles occur outside of $2 r^{*}$ (Fig. 3a). The pre-SEF composite mean has a statistically significantly higher tangential wind speed than the IN composite mean between $2 r^{*}$ and $6 r^{*}$, while the post-SEF group is statistically significantly higher than both the pre-SEF and IN groups outside of $2 r^{*}$. These signals in the pre- and post-SEF groups reflect the broadening of the outer wind field, which previous studies indicate is a precursor to secondary eyewall formation (Fudeyasu and Wang 2011; Fang and Zhang 2012; Rozoff et al. 2012; Didlake and Houze 2013b). As shown in Fig. 2, the radius of the secondary eyewall $\left(r_{2}\right)$ occurs anywhere from 40 to $120 \mathrm{~km}$. The radius of the secondary eyewall emerges between $2 r^{*}$ and $8 r^{*}$ in more than $85 \%$ of cases analyzed here; this is a wider range than that observed by Sitkowski et al. (2011) and Rogers et al. (2013). For the pre-SEF group, this region is where the earliest broadening of the wind field occurred. For the post-SEF group, this region demonstrates the continued broadening of the outer wind field and the emergence of the secondary eyewall. Interpreting in the context of select SEF theories, this region also corresponds with the stagnation radius for vortex Rossby waves, which propagate outward from the TC center (Montgomery and Kallenbach 1997; Chen and Yau 2001; Wang 2002a,b; Corbosiero et al.2006). Additionally, the broadened wind field in pre-SEF storms likely extends into the boundary layer, where unbalanced dynamics can then generate localized convection and an eventual secondary eyewall (Huang et al. 2012; Abarca and Montgomery 2013). Though significant differences among the profiles are apparent, no clear secondary eyewall wind maximum is visible in the $r^{*}$ framework, as noted in section 2 .

The vertical vorticity composite-mean profiles reveal a ringlike structure of vorticity radially inward of the primary eyewall $\left(1 r^{*}\right)$ of all three groups (Fig. 3b). The reader should note that the vorticity profiles cannot be directly interpreted from the tangential wind profiles (Fig. 3a) due to the variation in $r_{1}$ values among the different groups (Fig. 2). For the IN cases, the ringlike vorticity structure is consistent with Martinez et al. (2017) and characterizes a dynamically unstable structure and a thermodynamically isolated eye (Kossin and Eastin 2001; Braun et al. 2006; Houze 2010; Martinez et al. 2017). The pre-SEF composite-mean profile is statistically significantly lower than both the post-SEF and IN composite means inside the primary eyewall, as well as between $1 r^{*}$ and $2 r^{*}$. Martinez et al. (2017) found that both intensifying and weakening major hurricanes had a vorticity ring, but the weakening TCs had smaller vorticity magnitudes. Meanwhile, steady-state storms did not have a vorticity ring in the eyewall, but rather a flatter vorticity profile, which they attributed to differing TC regimes as described by Kossin and Eastin (2001). Utilizing the intensity change definitions from Martinez et al. (2017), $45 \%$ (37\%) of TCs in the pre-SEF (post-SEF) group meet the criteria for intensifying, $3 \%(27 \%)$ are weakening, and $42 \%(27 \%)$ fall under steady state (the remaining $\sim 10 \%$ in each group spanned more than one intensity change classification during the observation 
time span). Martinez et al. (2017) found that steady-state TCs exhibited smaller vorticity magnitudes than intensifying and weakening TCs; the larger proportion of steady-state TCs in the pre-SEF group may account for its smaller vorticity magnitude, compared to the postSEF group. However, we have not precisely quantified the effects of the weakening TCs on the magnitude of the vorticity in each group. The vorticity differences outside of the primary eyewall are small, but it is clear that the post-SEF group has statistically significant higher vorticity between much of $1 r^{*}$ and $4 r^{*}$. This increased vorticity corresponds to the expanded wind field from Fig. 3 and the associated increase in curvature vorticity.

The axisymmetric angular momentum was calculated in cylindrical coordinates, given by

$$
M=r v+\frac{1}{2} f r^{2},
$$

where $r$ is the radius, $v$ is the tangential wind, and $f$ is the Coriolis parameter. To account for the strong radial dependence of absolute angular momentum, we normalized each leg by the value of $M$ at $1 r^{*}$ to calculate the nondimensional angular momentum $M^{*}$ as done by Martinez et al. (2017). We then composited the resulting set means and determined the radii with statistically significant differences (Fig. 3c). Inside $1 r^{*}$, the differences among the groups are small, which was also the case for dimensional $M$ (not shown). Outside of $1 r^{*}$, the $M^{*}$ profiles are consistent with their corresponding tangential wind profiles. The pre-SEF group had higher $M^{*}$ values than the IN group between $2 r^{*}$ and $6 r^{*}$, which corresponds with the radii where the broadening of the outer wind field was observed (Fig. 3a). Outside of $2 r^{*}$, the post-SEF composite-mean profile was statistically significantly higher than the other groups, consistent with the observed higher tangential wind speeds. The differences between groups suggest that $M$ surfaces are advected radially inward over time during SEF, consistent with the findings of Bell et al. (2012).

\section{b. Axisymmetric composite-mean thermodynamic structures}

The kinematic variables showed a clear progression from IN to pre-SEF to post-SEF groups outside of $2 r^{*}$ that is expected with structural changes during SEF. We now examine the thermodynamic variables for signatures that also indicate some progression leading up to SEF.

The composite-mean temperature profiles were similar among the three groups (Fig. 4a). We find maximum temperatures in the eyewall cloud at $1 r^{*}$. The eyewall also has the greatest negative radial temperature gradient, which is consistent with the maintenance of thermal wind balance for the strongest TC winds in the low levels. The post-SEF group has a higher compositemean temperature than the pre-SEF and IN groups from the center to $4 r^{*}$, but this difference was statistically significant only near $1-1.5 r^{*}$. This region could be experiencing adiabatic warming associated with increased subsidence between the two concentric eyewalls, or it could be experiencing diabatic heating due to a possible increase in the convection at flight level. Next, we examine moisture to assess these conjectures.

A comparison of the composite-mean specific humidity profiles reveals that the post-SEF group has a larger amount of moisture present between $2 r^{*}$ and $4 r^{*}$ (Fig. 4b). This difference compared to the pre-SEF group is statistically significant, and a smaller stretch of statistically significant differences occur compared to the IN group. Based on the distributions of eyewall radii, this region corresponds with frequent emergence of the secondary eyewall, which would explain the moisture differences at these radii. In the eyewall cloud, specific humidity decreases rapidly in all three groups; the postSEF group had the highest amount of moisture here as well. The difference between the pre-SEF and post-SEF composite-mean specific humidity profiles is statistically significant between $4 r^{*}$ and $6 r^{*}$. Rainband activity at these radii may explain this difference. Didlake and Houze (2013b) illustrated that the appearance of drier air tends to occur preferentially in the stratiform portion of the rainband complex prior to secondary eyewall formation. To assess the importance of this signature and its location in the rainband complex, we employ the framework relative to both eyewalls in section 4 .

Comparing relative humidity $(\mathrm{RH})$ profiles, the IN composite mean has higher $\mathrm{RH}$ values than the ERC composite means throughout much of the radial profile (Fig. 4c). The differences between the IN and both ERC profiles are statistically significant just outside of $1 r^{*}$, possibly indicating fewer clouds in this region for ERC storms. The lower RH values here would then be indicative of a moat appearing outside of the eyewall. The $\mathrm{RH}$ in IN storms is also statistically significantly higher than the pre-SEF profile between $5 r^{*}$ and $6 r^{*}$. The postSEF group coincides with the first emergence of the secondary eyewall. Thus, a moat would indeed be developing at this time, which likely explains the clear dip in RH just outside of $1 r^{*}$ for post-SEF storms. To assess the structural significance of this result, we will examine the ERC storms relative to the radius of the secondary eyewall in addition to the primary eyewall in section 4 .

The composite-mean equivalent potential temperature $\left(\theta_{e}\right)$ profiles contain a maximum of $\theta_{e}$ within the eyewall cloud (Fig. 4d). Parameter $\theta_{e}$ decreases with increasing radius as temperature and specific humidity 

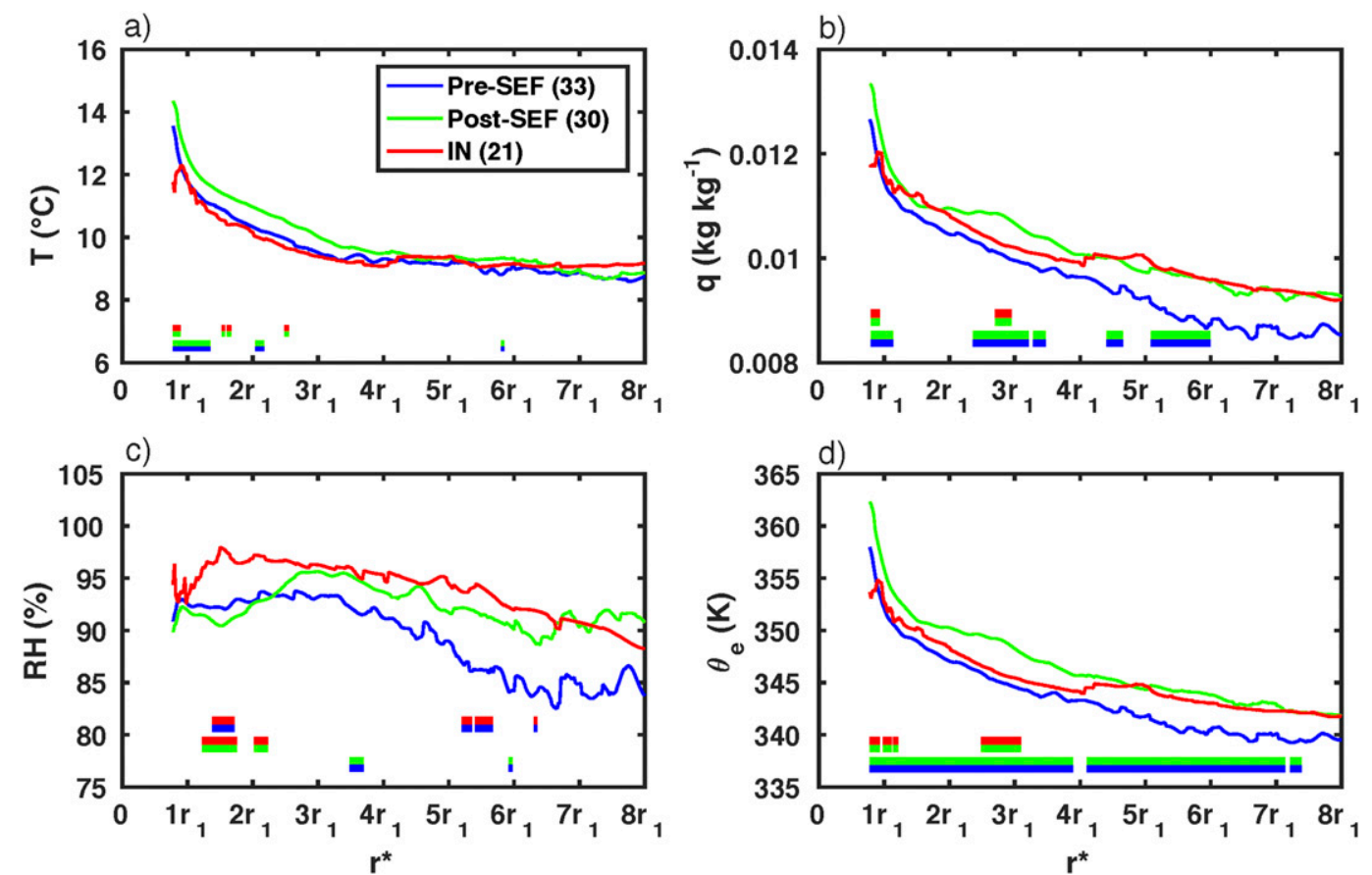

FIG. 4. As in Fig. 3, but for (a) temperature, (b) specific humidity, (c) RH, and (d) equivalent potential temperature.

both decrease. The difference between the pre- and post-SEF groups is statistically significant at nearly all radii between $1 r^{*}$ and $7 r^{*}$, primarily because of higher moisture content in the post-SEF group. This finding is consistent with several studies that show an increase in $\theta_{e}$ in the inner core of a storm undergoing SEF (e.g., Fang and Zhang 2012; Rozoff et al. 2012). The post-SEF group has a statistically significantly higher $\theta_{e}$ composite mean than the IN group between $2 r^{*}$ and $3 r^{*}$, corresponding with an increase in specific humidity likely associated with the emerging secondary eyewall.

Overall, the thermodynamic variables do not have as widespread a structural progression from IN to postSEF groups as that seen in the kinematic variables. Compared to the ERC storms, the IN storms only had notable differences in moisture at the primary eyewall, secondary eyewall, and moat. However, going from preto post-SEF, the composite storm had a clear increase in $\theta_{e}$ throughout most radii.

\section{Asymmetric composite structures of TCs undergoing secondary eyewall formation}

Using a normalization based on the radii of both the primary and secondary eyewalls, we are able to composite the ERC events in a way that highlights the importance of structures near the developing secondary eyewall. We now analyze observed asymmetric structures that occur prior to and during SEF. Specifically, we focus on the region surrounding $r_{2}$, where the secondary eyewall ultimately forms. Figure 5 plots the compositemean radial profiles in this framework, elucidating features that were smeared out by the normalization technique employed in section 3 . A clear secondary wind maximum at $r_{2}$ is evident in the post-SEF composite-mean profile, which is statistically significant, compared to the pre-SEF profile, which does not contain a secondary wind maximum. The lack of significant differences between the two profiles in the outer portion of the profile corroborates the result seen in section 3 that the broadening of the outer wind field is evident before a secondary wind maximum develops.

The composite-mean radial profiles of relative vorticity for pre-SEF and post-SEF in the $r^{* *}$ normalization (relative to the radii of both eyewalls) exhibit several significant differences (Fig. 6b). The differences in the vorticity maxima of the primary eyewall are a result of the different intensity of the mean profiles, illustrated in Fig. 6a. At $r_{2}$, the post-SEF composite-mean profile exhibits a secondary vorticity maximum associated with the developing secondary eyewall, which is statistically significantly different from the pre-SEF profile. If this vorticity maximum extended down to the boundary layer, it could serve as the convective vorticity anomaly (and thus, balanced vorticity gradient anomaly) that initiates a balanced dynamics feedback loop to amplify convection and enhance the developing secondary eyewall (Kepert 2013). 

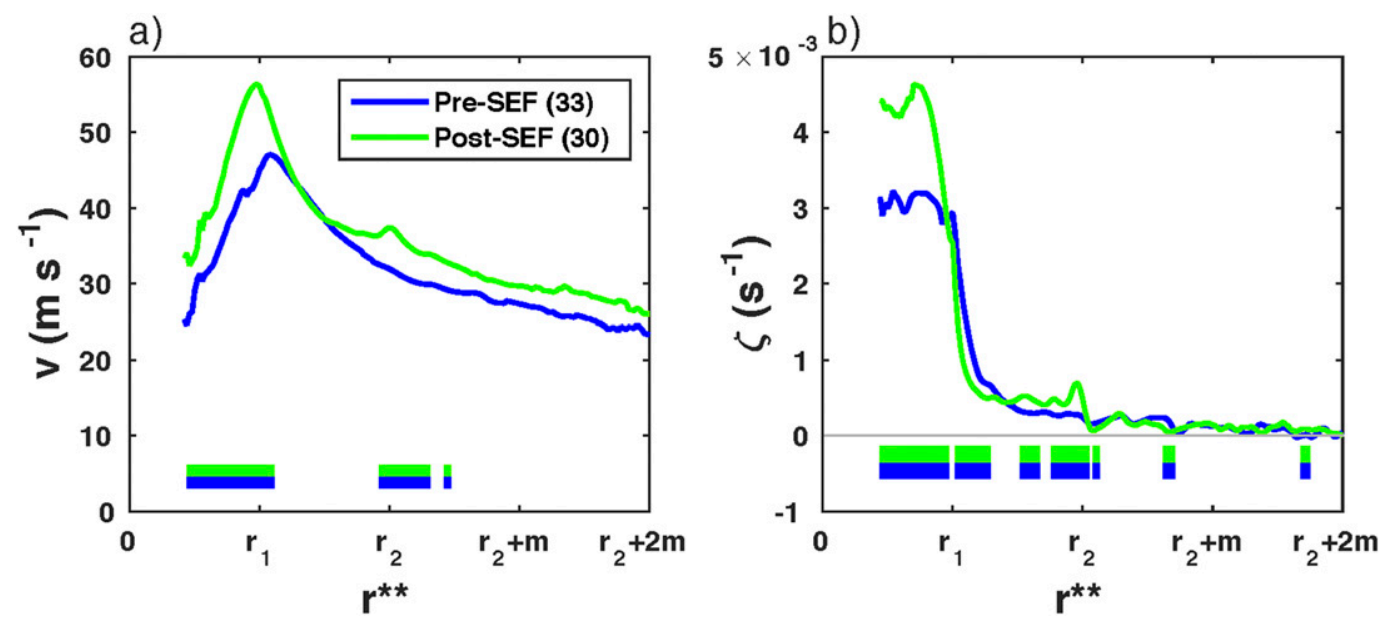

FIG. 5. Composite-mean radial profiles of (a) tangential wind and (b) relative vorticity for pre-SEF and post-SEF groups in $r^{* *}$ space (normalized by the radii of both the primary and secondary wind maxima). Numbers in the legend denote the sample count in each group.

These secondary wind and vorticity maxima were not evident in the analysis presented in section 3, but are well defined when this new normalization technique is employed. To further interpret how these features evolve during SEF, we continue using the $r^{* *}$ normalization technique to analyze the asymmetric evolution of the pre- and post-SEF groups. As discussed in section 2, we classify each flight leg into a quadrant based on the deep-layer environmental wind shear at the time of the flight leg to account for the role of shear in ERC evolution asymmetries.

\section{a. Quadrant-averaged kinematic structures}

Wavenumber-1 asymmetries in tropical cyclones can result from both deep-layer environmental wind shear and storm motion. Corbosiero and Molinari (2003) found that for both inner and outer regions of TCs, the wavenumber- 1 asymmetry aligns with the shear vector rather than the motion vector when the shear magnitude is moderate or large $\left(>5 \mathrm{~m} \mathrm{~s}^{-1}\right)$. To focus our quadrant analyses on shear-induced asymmetries, we present data from TCs experiencing shear greater than $5 \mathrm{~m} \mathrm{~s}^{-1}$ only.

Figure 6 presents the mean tangential winds within each quadrant. Between $r_{1}$ and $r_{2}$, the downshear quadrants have higher wind speeds than the upshear quadrants. Such downshear enhancement of tangential winds is different from previous composite studies who show a left-of-shear enhancement (Reasor et al. 2013; Rogers et al. 2013; Zhang et al. 2013; Uhlhorn et al. 2014), albeit these studies did not exclusively
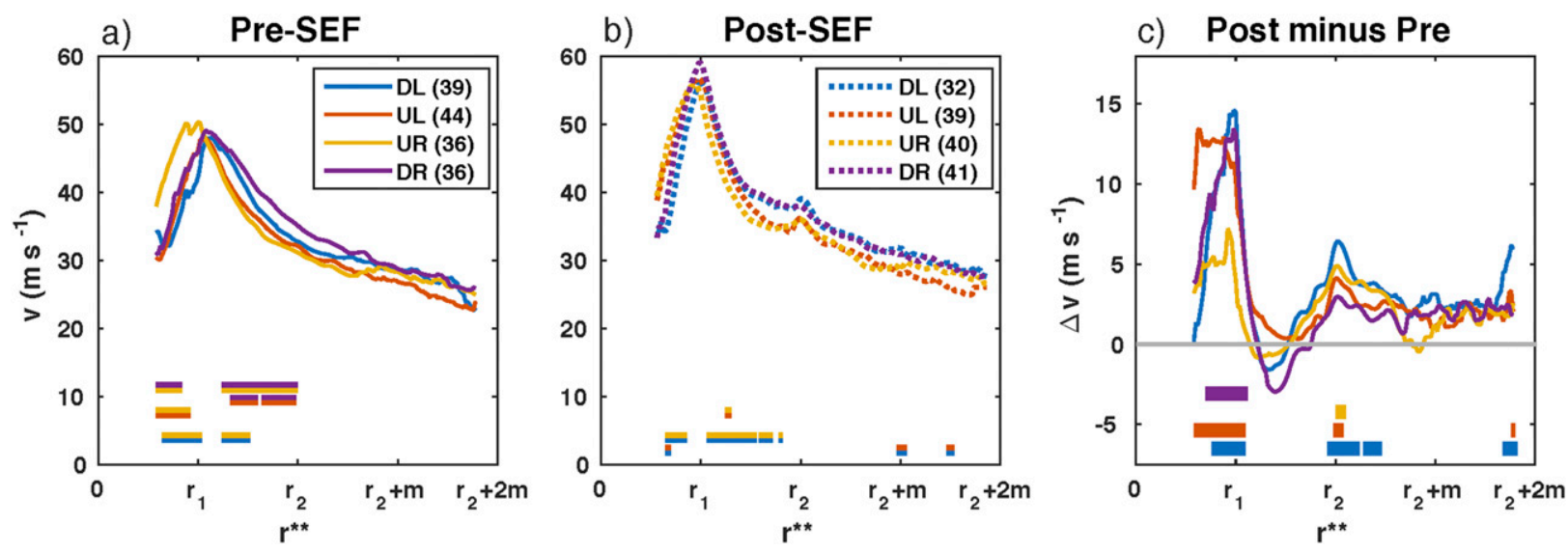

FIG. 6. (a) Quadrant mean profiles when shear magnitude is greater than $5 \mathrm{~m} \mathrm{~s}^{-1}$ for the pre-SEF group. Statistically significant differences between each quadrant pair are plotted along the bottom with colors corresponding to each quadrant. Numbers in the legend denote the sample count in each quadrant. (b) As in (a), but for the post-SEF group. (c) The change in tangential wind speed by quadrant between the post-SEF and pre-SEF groups. Statistically significant differences for the change in each quadrant are plotted along the bottom. 

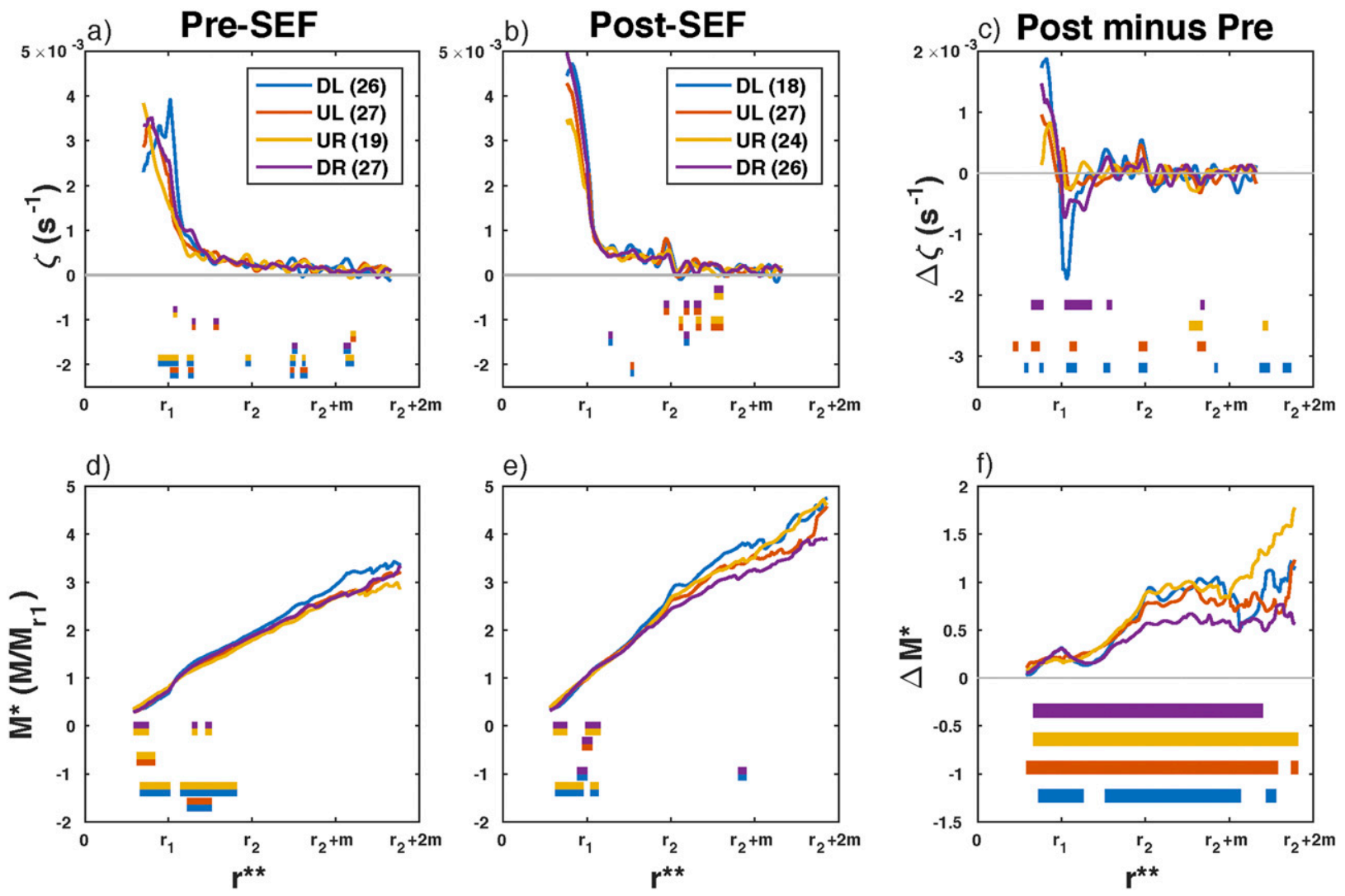

FIG. 7. As in Fig. 6, but for (a)-(c) vertical vorticity and (d)-(f) nondimensional absolute angular momentum.

focus on SEF cases. There are no statistically significant differences outside of $r_{2}$. These results suggest that the initial broadening of the outer wind field has the most notable asymmetry just outside of the primary eyewall.

For the post-SEF group, the extent of statistically significant differences decreases outside $r_{1}$, underscoring a reduction in the asymmetry inward of the secondary eyewall. We also note subtle differences in the wind profiles near $r_{2}$. Both DL and UL quadrants have peaks that are more prominent in their profiles. These are the only quadrants with statistically significant changes at $r_{2}$ from the pre- to post-SEF groups (Fig. 6c). This clear increase in peakedness suggests that SEF involves preferred strengthening of the $700-\mathrm{hPa}$ tangential winds in the left-of-shear quadrants. In an observational study of Hurricane Earl, Didlake et al. (2018) also found that the left-of-shear quadrants developed a clear secondary wind maximum first, which is consistent with the current composite analysis. Previous studies point to a left-of-shear preference for enhanced convective activity in developing and mature secondary eyewalls (Hence and Houze 2012a; Didlake et al. 2017). In comparison, the primary eyewall experiences the greatest change in tangential winds in the downshear quadrants, which is upwind of the observed secondary eyewall asymmetries. These differences in the arrangement of the two eyewalls are also consistent with previous studies (Hence and Houze 2012a; Didlake et al. 2017).

An assessment of relative vorticity shows a broad vorticity maximum in all quadrants in the eye and primary eyewall (Figs. 7a,b). The small regions suggesting statistical significance outside of the primary eyewall are likely false positive test results that can occur when applying statistical significance testing over a large number of radial points. In several of these regions, we have no a priori reason for differences to occur, and so we do not interpret the statistical significance signals as having implications on the TC structure.

In the post-SEF group, the secondary eyewall vorticity peak is most prominent in the left-of-shear quadrants (Fig. 7b), and at this radius, the left-of-shear quadrants have statistically significant increases going from pre- to post-SEF groups (Fig. 7c). These differences are consistent with the changes found in tangential wind.

The nondimensional angular momentum shows increasing momentum with radius in all quadrants (Fig. 7d). 
a) Pre-SEF

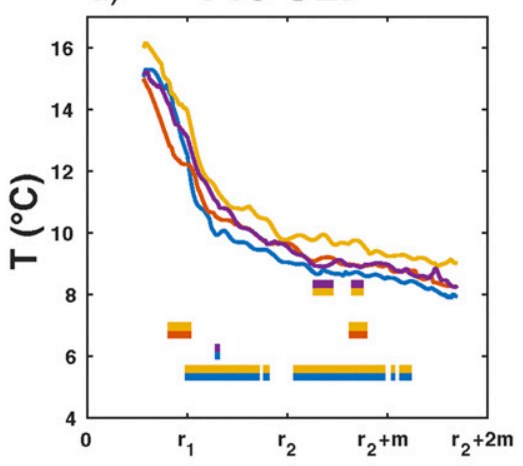

d)

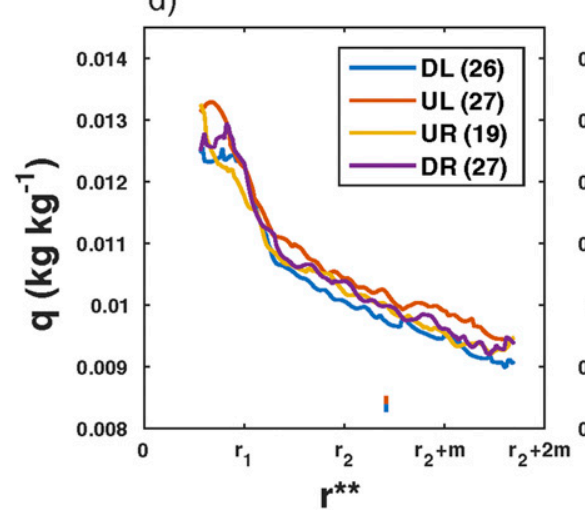

b) Post-SEF

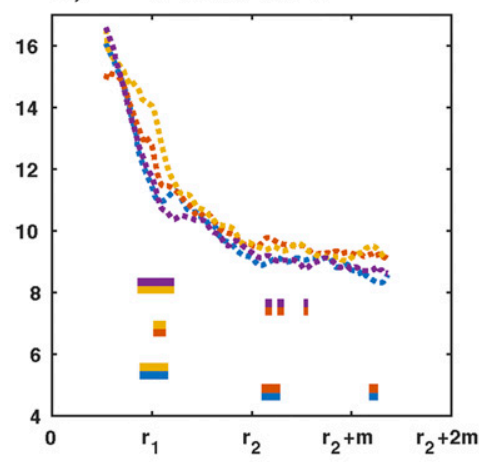

e)

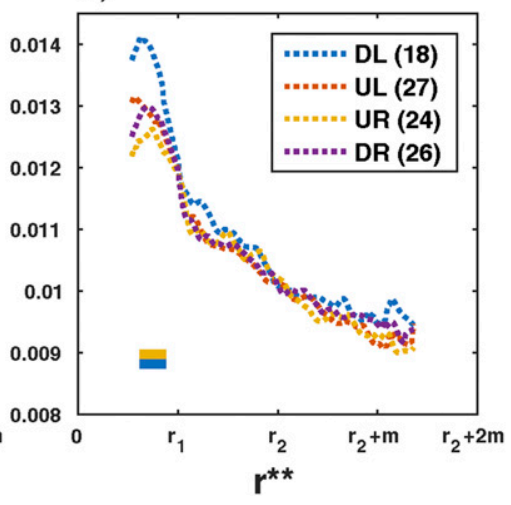

c) Post minus Pre
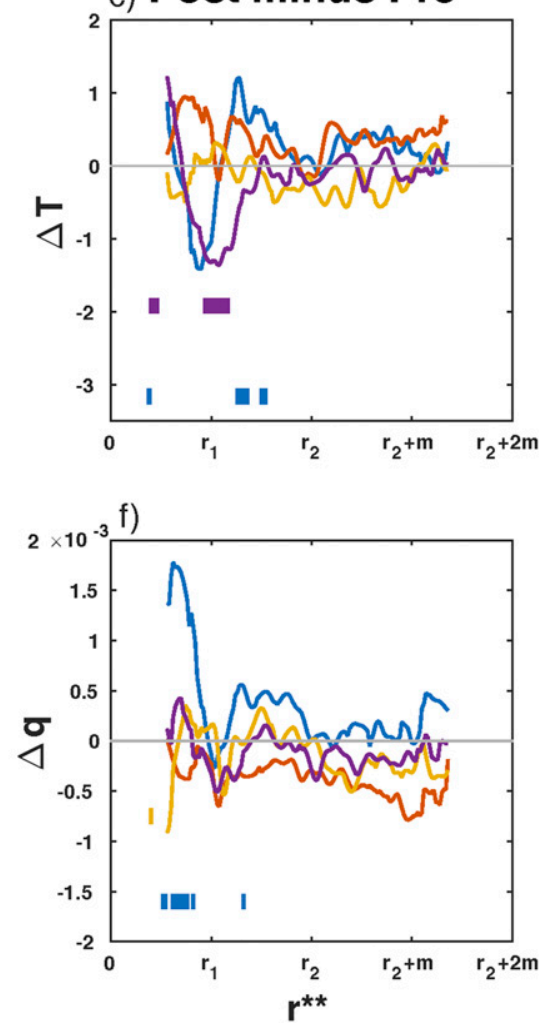

FIG. 8. As in Fig. 6, but for (a)-(c) temperature and (d)-(f) specific humidity.

In the pre-SEF group, the DL quadrant has higher angular momentum than the upshear quadrants between $r_{1}$ and $r_{2}$. While the broadening of the wind field between $r_{1}$ and $r_{2}$ was most notable in the DR and DL quadrants for tangential wind (Fig. 6a), the corresponding profile of enhanced angular momentum only appears in the DL quadrant. Though this enhancement is slight, its statistical significance through much of the region between $r_{1}$ and $r_{2}$ is unique to the pre-SEF group and could be dynamically important. The presence of a broad stratiform rainband may explain this signal, which would be consistent with Didlake and Houze (2013b), Qiu and Tan (2013), and Didlake et al. (2017, 2018). These previous studies found that the stratiform rainband, primarily located in the DL quadrant, induces mesoscale descending inflow that advects high angular momentum air inward from the surrounding environment. This inward advection could result in enhanced angular momentum values seen in the current observations.

After SEF, the similarity of the quadrant momentum values between $r_{1}$ and $r_{2}$ suggests that axisymmetrization has occurred (Fig. 7e). The momentum increased in all quadrants from the pre- to post-SEF groups (Fig. 7f), indicative of the locally increased tangential wind speeds associated with the secondary eyewall and broadened outer wind field.

\section{b. Quadrant-averaged thermodynamic structures}

Next, we analyze the thermodynamic mean quadrant profiles in the pre-SEF and post-SEF groups. The temperature profiles reveal a maximum in the eye and eyewall cloud, consistent with the presence of subsidence in the eye, and deep convection in the eyewall cloud (Houze 2010). Outside of $r_{1}$, the profiles decrease in both groups. The UR quadrant is statistically significantly warmer than the DL quadrant through much of $r_{1}$ to $r_{2}+m$ in the pre-SEF group (Fig. 8a), consistent with previous studies (Frank and Ritchie 1999; Zhang et al. 2013). These studies suggested that the difference between the UR and DL quadrants is due to precipitation and evaporative cooling in DL.

In the post-SEF group (Fig. 8b), the quadrant temperatures are relatively uniform. In particular, the DL is no longer cooler than UR throughout most of the profile. There are slight statistically significant differences near $r_{1}$ where the UR quadrant is slightly warmer than the other quadrants following SEF. This may be a result of more adiabatic warming from descent and the absence of cooling from evaporating precipitation in this 

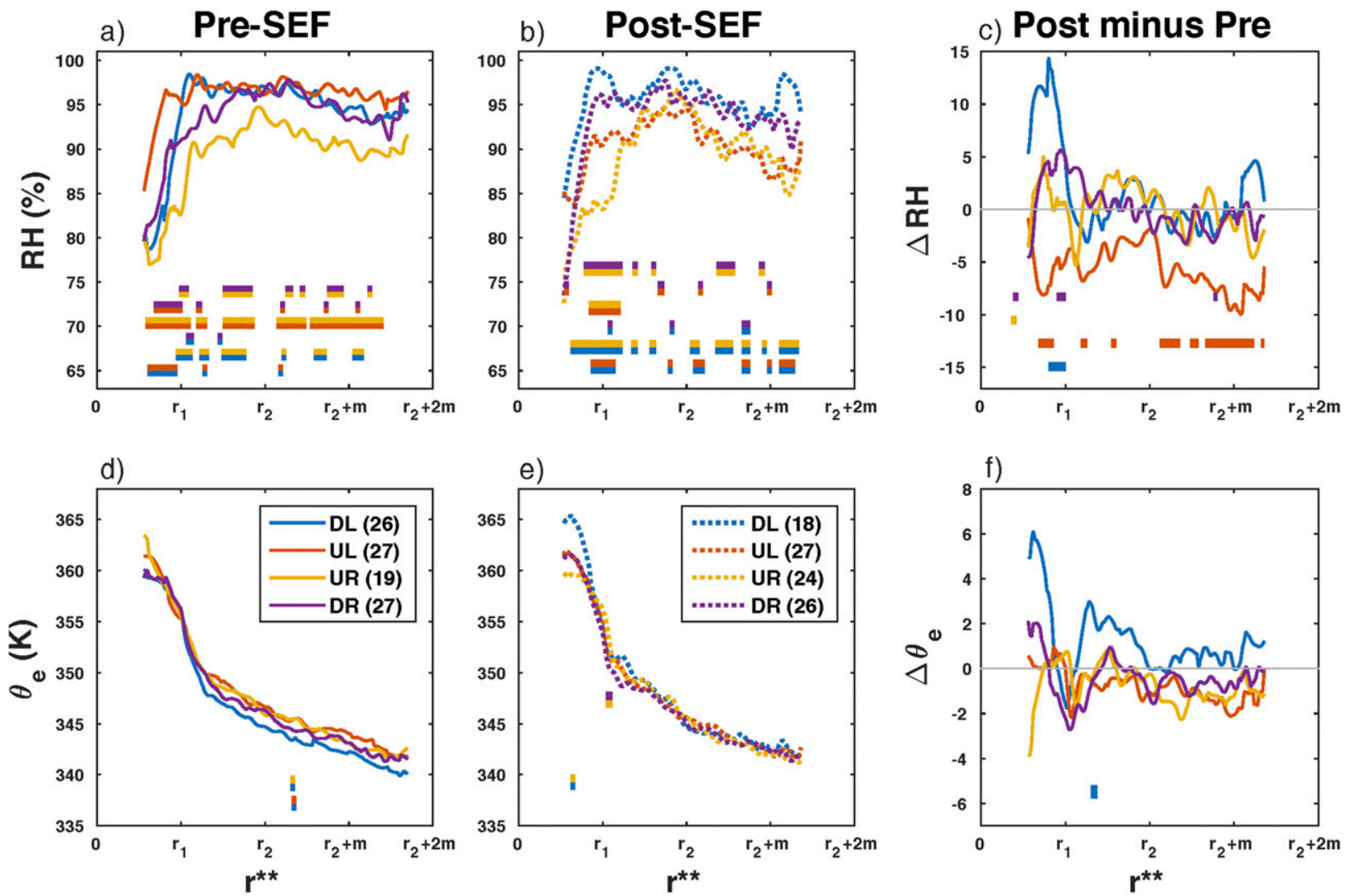

FIG. 9. As in Fig. 6, but for (a)-(c) RH and (d)-(f) equivalent potential temperature.

quadrant of the primary eyewall (Reasor et al. 2013; DeHart et al. 2014). The only statistically significant changes in temperature over time were between $r_{1}$ and $r_{2}$, where an increase (decrease) in the DL (DR) temperature occurred. The increase in the DL quadrant just outside the primary eyewall could be due to increasing subsidence as the moat forms, as this is also the quadrant with the most robust strengthening transverse circulation of the secondary eyewall (Didlake et al. 2017). The decrease in temperature at $r_{1}$ is likely a result of the contraction as higher temperatures associated with the DR eyewall convection move inward to smaller radii.

As in the axisymmetric profile, the specific humidity is at its maximum in the eyewall cloud and then decreases radially outward (Figs. 8d,e). An examination of preSEF specific humidity reveals no significant differences among the quadrants (Fig. 8d). The only post-SEF significant differences are between DL and UR in the eyewall cloud (Fig. 8e), which corresponds to a significant increase in DL specific humidity (Fig. 8f). This change may indicate an increased asymmetry in the primary eyewall convection and circulation as the secondary eyewall takes shape. The axisymmetric analysis showed an increase in specific humidity going from pre- to post-SEF throughout the profile. The quadrant composite analysis shows that the DL quadrant drives this increase for the inner eyewall, but the outer eyewall and rainband regions do not indicate that a specific quadrant dominates this increase.

The RH mean quadrant profiles have more statistically significant differences than all other thermodynamic variables (Figs. 9a,b). The observed regions of higher RH likely indicate increased clouds at flight level. In the pre-SEF group, the UL quadrant has higher RH radially inward of $r_{1}$, which indicates inward spiraling of the inner eyewall cloud in this quadrant. Radially outward, the UR quadrant is statistically significantly lower than the other quadrants at various points throughout the profile, which suggests decreased convection in this region. Between $r_{1}$ and $r_{2}$, axisymmetric $\mathrm{RH}$ analysis indicates possible moat development, compared to nonERC storms, and this decreasing cloud coverage appears to be most prevalent in the UR quadrant. Outside of $r_{2}$, lower RH in the UR indicates decreased rainband activity. This finding is generally consistent with several studies (e.g., Chen et al. 2006; Hence and Houze 2012b; Zawislak et al. 2016). The differences between UR and UL are statistically significant at many more radii than 
the differences between UR and the other quadrants. From this, we can infer that the broad cloud coverage of the rainband complex ends abruptly between the UL and UR quadrants and transitions to a dry, clear region. This result is consistent with the downwind end of a rainband complex comprising broad, stratiform precipitation and thus consistent cloud coverage in the UL quadrant (Didlake and Houze 2013b).

Immediately following SEF, DL consistently has the highest RH values throughout the profile. This difference is statistically significant, compared to the upshear quadrants, especially in the primary eyewall cloud where observations suggest the presence of a wavenumber-1 asymmetry in $\mathrm{RH}$ between the downshear and upshear regions of the storm. This wavenumber- 1 structure could be a manifestation of the effect that a sheared environment has on vertical motions in the upshear and downshear sides of an inner eyewall. Updrafts typically predominate the downshear quadrants, and downdrafts predominate the upshear quadrants (Jones 1995; Black et al. 2002; Eastin et al. 2005; Reasor et al. 2013; DeHart et al. 2014; Zawislak et al. 2016). This asymmetry manifests as higher (lower) relative humidity values in the downshear (upshear) quadrants. Following SEF, the secondary eyewall tends to act as a barrier that hinders low-level inflow of high- $\theta_{e}$ air from reaching the primary eyewall. Didlake et al. (2017) showed how this barrier effect occurs more in upshear quadrants, which may also contribute to the observed enhanced asymmetry in the primary eyewall seen in both $\mathrm{RH}$ and specific humidity.

Between $r_{1}$ and $r_{2}$, the reduction of $\mathrm{RH}$ becomes more apparent in the downshear profiles. The statistically significant differences between these quadrants and the UR quadrant occur less, which suggests that clearing of the moat region is reaching all quadrants. Outside of $r_{2}$, the UL quadrant no longer has a higher RH than the UR quadrant. This drying in UL is the most apparent RH change during SEF, as seen in statistically significant differences outside the secondary eyewall (Fig. 9c). As a result, the region outside $r_{2}$ has decreased symmetry in cloud coverage, just as in the inner eyewall. The transverse circulation of the strengthening secondary eyewall possibly triggered subsidence radially outward in the UL quadrant and decreased the RH here. Overall, the axisymmetric RH changes very little going from pre- to post-SEF, but the quadrant analysis shows that the asymmetry of this $\mathrm{RH}$ structure increases in both the inner eyewall and outside of the secondary eyewall, while the reduced $\mathrm{RH}$ in the moat becomes more axisymmetric.

An inspection of equivalent potential temperature $\theta_{e}$ reveals a maximum in the eye and eyewall cloud (Figs. 9d,e) and decreases radially outward similar to that observed in the temperature and specific humidity profiles. Despite differences in the temperature and RH profiles, there are no broad regions of statistically significant differences in either the pre- or post-SEF group. The axisymmetric analysis showed a clear increase in $\theta_{e}$ going from pre- to post-SEF in all regions of the TC, but the quadrant analysis shows that this increase is not particular to a specific quadrant.

\section{Conclusions}

Aircraft in situ observations at the 700-hPa level from FLIGHT+ (Vigh et al. 2016) were analyzed to assess the composite axisymmetric and asymmetric structures that are characteristic of secondary eyewall formation (SEF). We first examined the quasi evolution of axisymmetric TCs by comparing intensifying (IN) storms that did not undergo SEF to storms just prior to SEF (pre-SEF) and just after SEF (post-SEF). This axisymmetric composite analysis uses a radial framework that is normalized relative to the RMW in the primary eyewall. The SEF groups exhibit broadening of the wind field outside the primary eyewall in both tangential wind $v$ and nondimensional absolute angular momentum $\left(M^{*}\right)$ profiles that is not seen in the IN TCs, which was an expected structural change leading into SEF. Compared to IN TCs, the SEF groups develop distinct and expected thermodynamic differences that are characteristic of a concentric eyewall structure. The SEF groups have higher $\theta_{e}$ at the radii of both eyewalls that is associated with strengthening convection and lower relative humidity at the moat between the eyewalls that indicate decreasing clouds.

In our analysis using an RMW-relative normalization framework (the $r^{*}$ framework), features related to the secondary eyewall were smeared since the radii of the secondary eyewall varied widely. We therefore employed a different normalization technique, the $r^{* *}$ normalization, which takes into account the radii of both the primary eyewall $r_{1}$ and the secondary eyewall $r_{2}$. This framework successfully captured the axisymmetric secondary wind and vorticity maxima. Neither of these signals emerged in the often-used RMW-based normalization. With this new framework, we were able to perform a more thorough examination of features that are associated with SEF. This included an analysis of asymmetric features relative to the 850-200-hPa environmental wind shear.

In the pre-SEF group, kinematic and thermodynamic signatures corresponded well with the expected asymmetry of a mature rainband complex. The axisymmetric expansion of the wind field in the rainband regions corresponded to downshear enhancement of $v$ and downshear-left (DL) enhancement of $M^{*}$. Coinciding 
with the enhanced $M^{*}$, the DL quadrant often consists of stratiform rainband precipitation and mesoscale descending inflow, which locally increases angular momentum by advecting higher angular momentum air radially inward (Didlake and Houze 2013b). In the upshear-right (UR) quadrant, higher temperatures and lower relative humidity $(\mathrm{RH})$ were consistent with large-scale subsidence and limited rainband convection expected in this quadrant. In particular, the significant RH difference between the upshear-left (UL) and UR quadrants suggested an abrupt decline in $700-\mathrm{hPa}$ clouds, which is consistent with the downwind end of a mature rainband complex.

Going from pre-SEF to post-SEF, the tangential winds and vorticity at $r_{2}$ increase significantly in the leftof-shear quadrants and result in prominent secondary wind and vorticity maxima in these quadrants in the post-SEF group. The left-of-shear occurrence of these wind and vorticity peaks is consistent with Didlake et al. (2018), who hypothesized that the left-of-shear mesoscale descending inflow in stratiform rainband precipitation plays a primary role in spinning up an incipient secondary eyewall wind maximum. Meanwhile, the inner eyewall exhibits asymmetric tangential wind increases at $r_{2}$ that extend upwind from the outer eyewall. This composite result is consistent with concentric eyewall differences seen in Hence and Houze (2012a) and Didlake et al. (2017). These studies suggest that while the inner eyewall directly dynamically interacts with the environmental wind shear, the outer eyewall kinematic asymmetry likely results from interaction with the rainband complex.

The inner eyewall also became more asymmetric in its thermodynamic structure during SEF. This enhanced asymmetry is indicated by the drying and warming of the UR and moistening of the DL at $r_{1}$. This finding follows the expected asymmetry of vertical motion in the inner eyewall cloud, where updrafts dominate downshear quadrants and downdrafts dominate upshear (e.g., DeHart et al. 2014). It is also consistent with a weakening of the inner eyewall during an ERC due to the outer eyewall preventing sufficient low-level high- $\theta_{e}$ air from reaching the inner eyewall, as discussed by Didlake et al. (2017). While the axisymmetric RH profiles were similar between the pre- and post-SEF groups, the quadrant analysis of $\mathrm{RH}$ revealed that drying in the moat region appears first in the UR and then becomes more symmetric, a signal classically associated with concentric eyewalls.

Our results highlight the TC features and evolving structures that are ubiquitous during SEF. Additional work must be performed to analyze these kinematic and thermodynamic structures to determine their specific contribution to SEF in the context of proposed SEF theories. Additional reconnaissance and radar observations of ERCs will be useful in this endeavor. In addition, continued use of numerical models to examine the axisymmetric and asymmetric features leading to SEF will help us to gain a better understanding of the processes and thus improvement in forecasts of subsequent eyewall replacement cycles.

Acknowledgments. We thank Jonathan Vigh and his collaborators who developed the FLIGHT + dataset at NCAR, which was fundamental in the design of this study. In addition, we thank Fuqing Zhang, George Young, and Michael Bell for their valuable input during the progress of this research. We thank Jonathan Martinez for providing insight into FLIGHT + and information from his analysis. We also thank the two anonymous reviewers for providing comments that improved the manuscript. The Extended Flight Level Dataset for Tropical Cyclones (FLIGHT +) was created by the Research Applications Laboratory at the National Center for Atmospheric Research (NCAR) from data provided by the NOAA Hurricane Research Division of AOML and the U.S. Air Force Reserve. The creation of this dataset was initially funded through a grant from the Bermuda Institute of Ocean Sciences Risk Prediction Initiative (RPI2.0). NCAR is sponsored by the National Science Foundation. This research was supported by the NASA New Investigator Program (NIP) under Grant NNX16AI21G.

\section{REFERENCES}

Abarca, S. F., and M. T. Montgomery, 2013: Essential dynamics of secondary eyewall formation. J. Atmos. Sci., 70, 3216-3230, https://doi.org/10.1175/JAS-D-12-0318.1.

Bell, M. M., M. T. Montgomery, and W. C. Lee, 2012: An axisymmetric view of concentric eyewall evolution in Hurricane Rita (2005). J. Atmos. Sci., 69, 2414-2432, https://doi.org/ 10.1175/JAS-D-11-0167.1.

Black, M. L., and H. E. Willoughby, 1992: The concentric eyewall cycle of Hurricane Gilbert. Mon. Wea. Rev., 120, 947-957, https://doi.org/10.1175/1520-0493(1992)120<0947: $\mathrm{TCECOH}>2.0 . \mathrm{CO} ; 2$.

- J. F. Gamache, F. D. Marks, C. E. Samsury, and H. E. Willoughby, 2002: Eastern Pacific Hurricanes Jimena of 1991 and Olivia of 1994: The effect of vertical shear on structure and intensity. Mon. Wea. Rev., 130, 2291-2312, https://doi.org/ 10.1175/1520-0493(2002)130<2291:EPHJOA > 2.0.CO;2.

Braun, S. A., M. T. Montgomery, and Z. Pu, 2006: High-resolution simulation of Hurricane Bonnie (1998). Part I: The organization of eyewall vertical motion. J. Atmos. Sci., 63, 19-42, https://doi.org/10.1175/JAS3598.1.

Chen, S. S., J. A. Knaff, and F. D. Marks, 2006: Effects of vertical wind shear and storm motion on tropical cyclone rainfall asymmetries deduced from TRMM. Mon. Wea. Rev., 134, 3190-3208, https://doi.org/10.1175/MWR3245.1. 
Chen, Y., and M. K. Yau, 2001: Spiral bands in a simulated hurricane. Part I: Vortex Rossby wave verification. J. Atmos. Sci., 58, 2128-2145, https://doi.org/10.1175/1520-0469(2001)058<2128: SBIASH $>2.0 . \mathrm{CO} ; 2$.

Corbosiero, K. L., and J. Molinari, 2003: The relationship between storm motion, Vertical wind shear, and convective asymmetries in tropical cyclones. J. Atmos. Sci., 60, 366-376, https://doi.org/ 10.1175/1520-0469(2003)060<0366:TRBSMV>2.0.CO;2.

,,-- A. R. Aiyyer, and M. L. Black, 2006: The structure and evolution of Hurricane Elena (1985). Part II: Convective asymmetries and evidence for vortex Rossby waves. Mon. Wea. Rev., 134, 3073-3091, https://doi.org/10.1175/MWR3250.1.

Dai, Y., S. J. Majumdar, and D. S. Nolan, 2017: Secondary eyewall formation in tropical cyclones by outflow-jet interaction. J. Atmos. Sci., 74, 1941-1958, https://doi.org/10.1175/JAS-D16-0322.1.

DeHart, J. C., R. A. Houze Jr., and R. F. Rogers, 2014: Quadrant distribution of tropical cyclone inner-core kinematics in relation to environmental shear. J. Atmos. Sci., 71, 2713-2732, https://doi.org/10.1175/JAS-D-13-0298.1.

DeMaria, M., M. Mainelli, L. K. Shay, J. A. Knaff, and J. Kaplan, 2005: Further improvements to the Statistical Hurricane Intensity Prediction Scheme (SHIPS). Wea. Forecasting, 20, 531-543, https://doi.org/10.1175/WAF862.1.

Didlake, A. C., Jr., and R. A. Houze Jr., 2011: Kinematics of the secondary eyewall observed in Hurricane Rita (2005). J. Atmos. Sci., 68, 1620-1636, https://doi.org/10.1175/2011JAS3715.1.

_, and —, 2013a: Dynamics of the stratiform sector of a tropical cyclone rainband. J. Atmos. Sci., 70, 1891-1911, https://doi.org/10.1175/JAS-D-12-0245.1.

$\longrightarrow$, and - 2013b: Convective-scale variations in the innercore rainbands of a tropical cyclone. J. Atmos. Sci., 70, 504523, https://doi.org/10.1175/JAS-D-12-0134.1.

—, G. M. Heymsfield, P. D. Reasor, and S. R. Guimond, 2017: Concentric eyewall asymmetries in Hurricane Gonzalo (2014) observed by airborne radar. Mon. Wea. Rev., 145, 729-749, https://doi.org/10.1175/MWR-D-16-0175.1.

— P. D. Reasor, R. F. Rogers, and W.-C. Lee, 2018: Dynamics of the transition from spiral rainbands to a secondary eyewall in Hurricane Earl (2010). J. Atmos. Sci., 75, 2909-2929, https:// doi.org/10.1175/JAS-D-17-0348.1.

Dodge, P., R. W. Burpee, and F. D. Marks, 1999: The kinematic structure of a hurricane with sea level pressure less than 900 mb. Mon. Wea. Rev., 127, 987-1004, https://doi.org/10.1175/ 1520-0493(1999)127<0987:TKSOAH>2.0.CO;2.

Duchon, C. E., 1979: Lanczos filtering in one and two dimensions. J. Appl. Meteor., 18, 1016-1022, https://doi.org/10.1175/15200450(1979)018<1016:LFIOAT $>2.0$. CO;2.

Eastin, M. D., W. M. Gray, and P. J. Black, 2005: Buoyancy of convective vertical motions in the inner core of intense hurricanes. Part II : Case studies. Mon. Wea. Rev., 133, 209-227, https://doi.org/10.1175/MWR-2849.1.

Fang, J., and F. Zhang, 2012: Effect of beta shear on simulated tropical cyclones. Mon. Wea. Rev., 140, 3327-3346, https:// doi.org/10.1175/MWR-D-10-05021.1.

Frank, W. M., and E. A. Ritchie, 1999: Effects of environmental flow upon tropical cyclone structure. Mon. Wea. Rev., 127, 2044-2061, https://doi.org/10.1175/1520-0493(1999)127<2044: EOEFUT>2.0.CO;2.

Fudeyasu, H., and Y. Wang, 2011: Balanced contribution to the intensification of a tropical cyclone simulated in TCM4: Outer-core spinup process. J. Atmos. Sci., 68, 430-449, https:// doi.org/10.1175/2010JAS3523.1.
Gray, W. M., 1968: Global view of the origin of tropical disturbances and storms. Mon. Wea. Rev., 96, 669-700, https://doi.org/ 10.1175/1520-0493(1968)096<0669:GVOTOO>2.0.CO;2.

Hawkins, J., and M. Helveston, 2008: Tropical cyclone multiple eyewall characteristics. 28th Conf. on Hurricanes and Tropical Meteorology, Orlando, FL, Amer. Meteor. Soc., 14B.1, https://ams.confex.com/ams/28Hurricanes/techprogram/paper_ 138300.htm.

$\longrightarrow,-$, T. F. Lee, F. J. Turk, K. Richardson, C. Sampson, J. Kent, and R. Wade, 2006: Tropical cyclone multiple eyewall configurations. 27th Conf. on Hurricanes and Tropical Meteorology, Monterey, CA, Amer. Meteor. Soc., 6B.1, https://ams.confex.com/ ams/27Hurricanes/techprogram/paper_108864.htm.

Hence, D. A., and R. A. Houze Jr., 2012a: Vertical structure of tropical cyclone rainbands as seen by the TRMM Precipitation Radar. J. Atmos. Sci., 69, 2644-2661, https://doi.org/10.1175/ JAS-D-11-0323.1.

$\longrightarrow$, and — 2012b: Vertical structure of tropical cyclones with concentric eyewalls as seen by the TRMM Precipitation Radar. J. Atmos. Sci, 69, 1021-1036, https://doi.org/10.1175/JAS-D-11-0119.1.

Houze, R. A., Jr., 2004: Mesoscale convective systems. Rev. Geophys., 42, RG4003, https://doi.org/10.1029/2004RG000150.

_ 2010: Clouds in tropical cyclones. Mon. Wea. Rev., 138, 293 344, https://doi.org/10.1175/2009MWR2989.1.

— S. S. Chen, B. F. Smull, W. C. Lee, and M. M. Bell, 2007: Hurricane intensity and eyewall replacement. Science, 315, 1235-1239, https://doi.org/10.1126/science.1135650.

Huang, Y.-H., M. T. Montgomery, and C.-C. Wu, 2012: Concentric eyewall formation in Typhoon Sinlaku (2008). Part II: Axisymmetric dynamical processes. J. Atmos. Sci., 69, 662-674, https://doi.org/10.1175/JAS-D-11-0114.1.

Jones, S. C., 1995: The evolution of vortices in vertical shear. I: Initially barotropic vortices. Quart. J. Roy. Meteor. Soc., 121, 821-851, https://doi.org/10.1002/qj.49712152406.

Kepert, J. D., 2013: How does the boundary layer contribute to eyewall replacement cycles in axisymmetric tropical cyclones? J. Atmos. Sci., 70, 2808-2830, https://doi.org/10.1175/JAS-D13-046.1.

Kossin, J. P., and M. D. Eastin, 2001: Two distinct regimes in the kinematic and thermodynamic structure of the hurricane eye and eyewall. J. Atmos. Sci., 58, 1079-1090, https://doi.org/ 10.1175/1520-0469(2001)058<1079:TDRITK > 2.0.CO;2.

_ , and M. Sitkowski, 2009: An objective model for identifying secondary eyewall formation in hurricanes. Mon. Wea. Rev., 137, 876-892, https://doi.org/10.1175/2008MWR2701.1.

Martinez, J., M. M. Bell, J. L. Vigh, and R. F. Rogers, 2017: Examining tropical cyclone structure and intensification with the FLIGHT+ Dataset from 1999 to 2012. Mon. Wea. Rev., 145, 4401-4421, https://doi.org/10.1175/MWR-D-17-0011.1.

Montgomery, M. T., and R. J. Kallenbach, 1997: A theory for vortex Rossby-waves and its application to spiral bands and intensity changes in hurricanes. Quart. J. Roy. Meteor. Soc., 123, 435-465, https://doi.org/10.1002/qj.49712353810.

Parker, M. D., and R. H. Johnson, 2000: Organizational modes of midlatitude mesoscale convective systems. Mon. Wea. Rev., 128, 3413-3436, https://doi.org/10.1175/1520-0493(2001) $129<3413$ :OMOMMC $>2.0$.CO;2.

Qiu, X., and Z.-M. Tan, 2013: The roles of asymmetric inflow forcing induced by outer rainbands in tropical cyclone secondary eyewall formation. J. Atmos. Sci., 70, 953-974, https:// doi.org/10.1175/JAS-D-12-084.1.

Reasor, P. D., R. F. Rogers, and S. Lorsolo, 2013: Environmental flow impacts on tropical cyclone structure diagnosed from 
airborne Doppler radar composites. Mon. Wea. Rev., 141, 2949-2969, https://doi.org/10.1175/MWR-D-12-00334.1.

Rogers, R. F., P. D. Reasor, and S. Lorsolo, 2013: Airborne Doppler observations of the inner-core structural differences between intensifying and steady-state tropical cyclones. Mon. Wea. Rev., 141, 2970-2991, https://doi.org/10.1175/MWR-D12-00357.1.

Rozoff, C. M., W. H. Schubert, B. D. McNoldy, and J. P. Kossin, 2006: Rapid filamentation zones in intense tropical cyclones. J. Atmos. Sci., 63, 325-340, https://doi.org/10.1175/JAS3595.1.

_, D. S. Nolan, J. P. Kossin, F. Zhang, and J. Fang, 2012: The roles of an expanding wind field and inertial stability in tropical cyclone secondary eyewall formation. J. Atmos. Sci., 69, 2621-2643, https://doi.org/10.1175/JAS-D-11-0326.1.

Sitkowski, M., J. P. Kossin, and C. M. Rozoff, 2011: Intensity and structure changes during hurricane eyewall replacement cycles. Mon. Wea. Rev., 139, 3829-3847, https://doi.org/10.1175/ MWR-D-11-00034.1.

,,--- , and J. A. Knaff, 2012: Hurricane eyewall replacement cycle thermodynamics and the relict inner eyewall circulation. Mon. Wea. Rev., 140, 4035-4045, https://doi.org/ 10.1175/MWR-D-11-00349.1.

Sun, Y. Q., Y. Jiang, B. Tan, and F. Zhang, 2013: The governing dynamics of the secondary eyewall formation of Typhoon Sinlaku (2008). J. Atmos. Sci., 70, 3818-3837, https://doi.org/ 10.1175/JAS-D-13-044.1.

Tang, X., Z.-M. Tan, J. Fang, Y. Q. Sun, and F. Zhang, 2017: Impact of the diurnal radiation cycle on secondary eyewall formation. J. Atmos. Sci., 74, 3079-3098, https://doi.org/10.1175/ JAS-D-17-0020.1.

Terwey, W. D., and M. T. Montgomery, 2008: Secondary eyewall formation in two idealized, full-physics modeled hurricanes. J. Geophys. Res., 113, D12112, https://doi.org/10.1029/ 2007JD008897.

Uhlhorn, E. W., B. W. Klotz, T. Vukicevic, P. D. Reasor, and R. F. Rogers, 2014: Observed hurricane wind speed asymmetries and relationships to motion and environmental shear. Mon. Wea. Rev., 142, 1290-1311, https://doi.org/10.1175/ MWR-D-13-00249.1.

Vigh, J. L., and Coauthors, 2016: FLIGHT+: The Extended Flight Level Dataset for Tropical Cyclones (Version 1.1). Tropical Cyclone Data Project, National Center for Atmospheric
Research, Research Applications Laboratory, Boulder, CO, accessed 17 October 2017, https://doi.org/10.5065/D6WS8R93.

Wang, H., C.-C. Wu, and Y. Wang, 2016: Secondary eyewall formation in an idealized tropical cyclone simulation: Balanced and unbalanced dynamics. J. Atmos. Sci., 73, 3911-3930, https://doi.org/10.1175/JAS-D-15-0146.1.

Wang, Y., 2002a: Vortex Rossby waves in a numerically simulated tropical cyclone. Part I: Overall structure, potential vorticity, and kinetic energy budgets. J. Atmos. Sci., 59, 1213-1238, https://doi.org/10.1175/1520-0469(2002)059<1213:VRWIAN > 2.0. $\mathrm{CO} ; 2$.

, 2002b: Vortex Rossby waves in a numerically simulated tropical cyclone. Part II: The role in tropical cyclone structure and intensity changes. J. Atmos. Sci., 59, 1239-1262, https:// doi.org/10.1175/1520-0469(2002)059<1239:VRWIAN>2.0.CO;2.

Willoughby, H. E., and M. B. Chelmow, 1982: Objective determination of hurricane tracks from aircraft observations. Mon. Wea. Rev., 110, 1298-1305, https://doi.org/10.1175/1520-0493(1982) $110<1298$ :ODOHTF $>2.0 . \mathrm{CO} ; 2$

_ J. A. Clos, and M. G. Shoreibah, 1982: Concentric eye walls, secondary wind maxima, and the evolution of the hurricane vortex. J. Atmos. Sci., 39, 395-411, https://doi.org/10.1175/ 1520-0469(1982)039<0395:CEWSWM >2.0.CO;2.

Zawislak, J., H. Jiang, G. R. Alvey, E. J. Zipser, R. F. Rogers, J. A. Zhang, and S. N. Stevenson, 2016: Observations of the structure and evolution of Hurricane Edouard (2014) during intensity change. Part I: Relationship between the thermodynamic structure and precipitation. Mon. Wea. Rev., 144, 3333-3354, https://doi.org/10.1175/MWR-D-16-0018.1.

Zhang, F., D. Tao, Y. Q. Sun, and J. D. Kepert, 2017: Dynamics and predictability of secondary eyewall formation in sheared tropical cyclones. J. Adv. Model. Earth Syst., 9, 89-112, https:// doi.org/10.1002/2016MS000729.

Zhang, J. A., R. F. Rogers, P. D. Reasor, E. W. Uhlhorn, and F. D. Marks, 2013: Asymmetric hurricane boundary layer structure from dropsonde composites in relation to the environmental vertical wind shear. Mon. Wea. Rev., 141, 3968-3984, https:// doi.org/10.1175/MWR-D-12-00335.1.

Zipser, E. J., R. J. Meitín, and M. A. LeMone, 1981: Mesoscale motion fields associated with a slowly moving GATE convective band. J. Atmos. Sci., 38, 1725-1750, https://doi.org/ 10.1175/1520-0469(1981)038<1725:MMFAWA > 2.0.CO;2. 